\title{
Multivariable Wilson polynomials and degenerate Hecke algebras
}

\author{
Wolter Groenevelt
}

\begin{abstract}
We study a rational version of the double affine Hecke algebra associated to the nonreduced affine root system of type $\left(C_{n}^{\vee}, C_{n}\right)$. A certain representation in terms of difference-reflection operators naturally leads to the definition of nonsymmetric versions of the multivariable Wilson polynomials. Using the degenerate Hecke algebra we derive several properties, such as orthogonality relations and quadratic norms, for the nonsymmetric and symmetric multivariable Wilson polynomials.
\end{abstract}

Mathematics Subject Classification (2000). Primary 33C52, 33C80;

Secondary 20C08.

\section{Introduction}

The Macdonald theory of multivariable orthogonal polynomials associated to root systems can be understood using a specific representation of Cherednik's double affine Hecke algebra (DAHA) in terms of $q$-difference-reflection operators, see e.g. Cherednik [2] and Macdonald [12]. Koornwinder polynomials [11] generalize the Macdonald polynomials associated to classical root systems. The algebraic structure underlying the Koornwinder polynomials is Sahi's DAHA associated to the nonreduced affine root system of type $\left(C_{n}^{\vee}, C_{n}\right)$, see Noumi [14], Sahi [16,17], and Stokman [18]. The Koornwinder polynomials depend, besides the base $q$, on five parameters, corresponding to the number of $W$-orbits in the affine root system of type $\left(C_{n}^{\vee}, C_{n}\right)$ with corresponding affine Weyl group $W$. Many families of multivariable orthogonal polynomials can be obtained as limits of the Koornwinder polynomials, see e.g. [21] and [19].

The author likes to thank Jasper Stokman for stimulating discussions. Parts of this research were done at the Korteweg-De Vries Institute for Mathematics at the University of Amsterdam, while supported by the Netherlands Organization for Scientific Research (NWO) for the Vidi-project "Symmetry and modularity in exactly solvable models". 
Several of these families have been associated in the literature to degenerate versions of double affine Hecke algebras. For instance, the Heckman-Opdam polynomials [10] are naturally associated to a trigonometric degenerate DAHA [15].

In this paper, we study an algebra $\mathcal{H}$ which is a rational degeneration of the DAHA of type $\left(C_{n}^{\vee}, C_{n}\right)$, and we show that multivariable Wilson polynomials are associated to $\mathcal{H}$ in a natural way. Multivariable Wilson polynomials are limits of the Koornwinder polynomials (for $q \rightarrow 1$ ) depending on five limiting parameters, see van Diejen [20,21]. The one-variable Wilson polynomials [22] are the most general orthogonal polynomials of hypergeometric type, i.e., all hypergeometric orthogonal polynomials, for instance, the Jacobi polynomials, can be obtained as a limit of the one-variable Wilson polynomials. It may be expected that the multivariable Wilson polynomials play a similar role in the theory of orthogonal polynomials of hypergeometric type associated to root systems. Let us show that Wilson polynomials formally generalize the $B C$-type Heckman-Opdam polynomials. Under suitable conditions on the parameters the multivariable Wilson polynomials are orthogonal on $\left(i \mathbb{R}_{+}\right)^{n}$ with respect to the weight function

$$
\Delta_{+}(x)=\prod_{1 \leq j<k \leq n} \frac{\Gamma\left(t \pm x_{j} \pm x_{k}\right)}{\Gamma\left( \pm x_{j} \pm x_{k}\right)} \prod_{j=1}^{n} \frac{\Gamma\left(a \pm x_{j}\right) \Gamma\left(b \pm x_{j}\right) \Gamma\left(c \pm x_{j}\right) \Gamma\left(d \pm x_{j}\right)}{\Gamma\left( \pm 2 x_{j}\right)} .
$$

Here we use the notation $\Gamma(\alpha \pm \beta)=\Gamma(\alpha+\beta) \Gamma(\alpha-\beta)$. The weight function $\Delta_{+}(x)$ may be considered as a generalization of the weight function for the $B C$-type Heckman-Opdam polynomials. Indeed, divide by a factor

$$
\begin{aligned}
& \prod_{j=1}^{n} \Gamma(a+c+(j-1) t) \Gamma(b+c+(j-1) t) \\
& \quad \times \Gamma(a+d+(j-1) t) \Gamma(b+d+(j-1) t),
\end{aligned}
$$

and substitute

$$
a=b=\frac{\alpha+1}{2}, \quad c=\frac{\beta+1}{2}+\mathrm{i} \gamma, \quad d=\frac{\beta+1}{2}-\mathrm{i} \gamma, \quad x_{j}=\mathrm{i} \gamma \sqrt{y_{j}},
$$

then after applying Stirling's formula we find that in the limit $\gamma \rightarrow \infty$,

$$
\Delta_{+}(x) \sim \frac{\sqrt{y_{1} \cdots y_{n}}}{\gamma^{n}} \prod_{1 \leq j<k \leq n}\left|y_{j}-y_{k}\right|^{2 t} \prod_{j=1}^{n} y_{j}^{\alpha}\left(1-y_{j}\right)^{\beta}, \quad y_{1}, \ldots, y_{n} \in(0,1),
$$

and $\Delta_{+}(x)$ vanishes exponentially if $y_{j}>1$ for any $j$.

The rational DAHA $\mathcal{H}$ that we study has a faithful representation in terms of difference-reflection operators, much like the representation of the trigonometric DAHA from [1]. Nonsymmetric versions of the multivariable Wilson polynomials then appear in the representation theory of $\mathcal{H}$ as the polynomial eigenfunctions of the analogues of the Cherednik operators. Methods from double affine Hecke algebras, see e.g. [2,12], can now be used to 
obtain properties of the nonsymmetric multivariable Wilson polynomials, such as orthogonality relations and a duality property. The rank one version of the algebra $\mathcal{H}$ and the corresponding one-variable Wilson polynomials have been studied in [8]. Let us remark that a four-parameter subfamily of the (symmetric) multivariable Wilson polynomials have been obtained by Zhang [23] in the context of degenerate Hecke algebras as the Harish-Chandra transform of the $B C$-type Heckman-Opdam polynomials. This shows that for suitable parameters the multivariable Wilson polynomials, multiplied by certain $\Gamma$-functions, form an orthogonal basis of the $L^{2}$-space consisting of functions on $\mathbb{R}^{n}$ with the Harish-Chandra measure $|c(\lambda)|^{-2} d \lambda$. It would be interesting to see if Zhang's results can be extended to the nonsymmetric case.

The algebra $\mathcal{H}$ has appeared earlier in the literature; it is isomorphic to a rational generalized DAHA defined by Etingof et al. [3]. Results in [3] then imply that $\mathcal{H}$ is also closely related to several other algebras appearing in the literature, e.g. $\mathcal{H}$ is the spherical subalgebra of a certain deformed preprojective algebra by Gan and Ginzburg [7]. In this paper we obtain several new properties of the algebra $\mathcal{H}$. One remarkable property is that it contains a subalgebra $H$ that may be considered as a deformation of $\mathbb{C}[W]$ with deformed braid-relations. To be more precise, as an algebra $H$ is generated by $T_{0}, \ldots, T_{n}$, which satisfy the following braid-type relations:

$$
\begin{aligned}
T_{i} T_{i+1} T_{i} T_{i+1}+t T_{i} T_{i+1} & =T_{i+1} T_{i} T_{i+1} T_{i}+t T_{i+1} T_{i}, \quad i=0, n-1, \\
T_{i} T_{i+1} T_{i} & =T_{i+1} T_{i} T_{i+1}, \quad i \in[1, n-2] \\
T_{i} T_{j} & =T_{j} T_{i}, \quad|i-j| \geq 2
\end{aligned}
$$

Here $t$ is some complex parameter. For $t=0$ the above relations are the usual braid relations for $W$. Deformations like this, i.e. deformations of a group algebra $\mathbb{C}[G], G$ a Coxeter group, with deformed braid relations, have recently been studied by Etingof and Rains $[5,6]$.

The paper is organized as follows. After some preliminaries on root systems, we define in Sect. 2 a subalgebra $H$ of the trigonometric DAHA $\mathfrak{H}$, and it is shown that the subalgebra $H$ is a deformation of the group algebra $\mathbb{C}[W]$. Then in Sect. 3 we define the rational DAHA $\mathcal{H}$ as a subalgebra of $\mathfrak{H}$ that is generated by $H$ and a polynomial algebra. We derive several useful properties of $\mathcal{H}$, such as a PBW-theorem, and we define the analogues of Cherednik's $Y$-operators. In Sect. 4 we study a faithful representation $\pi$ of $\mathcal{H}$ in terms of difference-reflection operators on the vector space $\mathcal{P}$ consisting of polynomials in $n$ variables. The nonsymmetric multivariable Wilson polynomials are used to describe the decomposition of $\mathcal{P}$ into irreducible $\pi(H)$-modules. In Sect. 5 we derive several properties of the nonsymmetric Wilson polynomials. Finally, in Sect. 6 we show how well-known properties of the (symmetric) multivariable Wilson polynomials can be obtained from the representation theory of $\mathcal{H}$. 


\section{The degenerate affine Hecke algebra}

In this section we define an algebra $H$ that we consider as a degenerate affine Hecke algebra of type $\widetilde{C}_{n}$. Throughout the paper we use the following notation: for integers $m, n$ with $m<n$ we write $[m, n]$ for the set $\{m, m+1, \ldots, n\}$.

\subsection{The affine root system $\left(C_{n}^{\vee}, C_{n}\right)$}

We fix an integer $n \geq 2$. Let $\left\{\epsilon_{j}\right\}_{j=1}^{n}$ be the standard orthonormal basis for $V=\mathbb{R}^{n}$ with standard inner product $\langle\cdot, \cdot\rangle$. Let $\widehat{V}$ denote the vector space of affine linear transformations from $V$ to $\mathbb{R}$. We identify $\widehat{V}$ with $V \oplus \mathbb{R} \delta$ by writing $f \in \widehat{V}$ as

$$
f(u)=\langle v, u\rangle+c \delta(u), \quad u \in V,
$$

for some $v \in V$ and $c \in \mathbb{R}$, where $\delta: V \rightarrow \mathbb{R}$ is the constant function defined by $\delta(u)=1$ for all $u \in V$. The inner product on $V$ is extended to a bilinear form on $\widehat{V}$ by

$$
\left\langle\lambda_{1}+\mu_{1} \delta, \lambda_{2}+\mu_{2} \delta\right\rangle=\left\langle\lambda_{1}, \lambda_{2}\right\rangle, \quad \lambda_{1}, \lambda_{2} \in V, \quad \mu_{1}, \mu_{2} \in \mathbb{R} .
$$

We define

$$
a_{0}=\delta-2 \epsilon_{1}, \quad a_{i}=\epsilon_{i}-\epsilon_{i+1}(i=1, \ldots, n-1), \quad a_{n}=2 \epsilon_{n} .
$$

The elements $a_{i} \in \widehat{V}$ are the simple roots for the affine root system of type $\widetilde{C}_{n}$. Let $W$ be the subgroup of $\operatorname{GL}(\widehat{V})$ generated by the simple reflections $s_{a_{i}}=s_{i}$, $i=0, \ldots, n$, where for $\beta \in \widehat{V}$ with $\langle\beta, \beta\rangle \neq 0$ the reflection $s_{\beta}$ is defined by

$$
s_{\beta} f=f-\left\langle f, \beta^{\vee}\right\rangle \beta, \quad \beta^{\vee}=\frac{2 \beta}{\langle\beta, \beta\rangle},
$$

for $f \in \widehat{V}$. The group $W$ is a Coxeter group with relations $s_{i}^{2}=1, i \in[0, n]$, and

$$
\begin{aligned}
s_{i} s_{i+1} s_{i} s_{i+1} & =s_{i+1} s_{i} s_{i+1} s_{i}, \quad i=0, n-1, \\
s_{i} s_{i+1} s_{i} & =s_{i+1} s_{i} s_{i+1}, \quad i \in[1, n-2], \\
s_{i} s_{j} & =s_{j} s_{i}, \quad i, j \in[0, n],|i-j| \geq 2 .
\end{aligned}
$$

The set $\mathcal{R}_{r}=W\left\{a_{0}, \ldots, a_{n}\right\} \subset \widehat{V}$ is the (reduced) affine root system of type $\widetilde{C}_{n}$. The nonreduced affine root system of type $\left(C_{n}^{\vee}, C_{n}\right)$ is the root system $\mathcal{R}=\mathcal{R}_{r}^{\vee} \cup \mathcal{R}_{r}$. The root systems $\mathcal{R}, \mathcal{R}_{r}^{\vee}$ and $\mathcal{R}_{r}$ all have $W$ as corresponding affine Weyl group. We denote by $W_{0} \subset W$ the (finite) Weyl group generated by $s_{1}, \ldots, s_{n}$. Note that the subgroup generated by $s_{1}, \ldots, s_{n-1}$ is isomorphic to the symmetric group $\mathcal{S}_{n}$. The set $\Sigma=W_{0}\left\{a_{1}, \ldots, a_{n}\right\}$ is the root system of type $C_{n}$. We write $\mathcal{R}^{ \pm}, \mathcal{R}_{r}^{ \pm}, \Sigma^{ \pm}$for the positive/negative roots in $\mathcal{R}, \mathcal{R}_{r}, \Sigma$, respectively, with respect to our choice of simple roots. In particular,

$$
\mathcal{R}_{r}^{+}=\Sigma^{+} \cup\left\{\alpha \in \mathcal{R}_{r} \mid \alpha(0)>0\right\} .
$$


The co-root lattice $Q^{\vee}$ and the weight lattice $P$ of $\Sigma$ in $V$ can both be identified with $\Lambda=\bigoplus_{i=1}^{n} \mathbb{Z} \epsilon_{i}$. We denote by $\Lambda^{+}$the cone of dominant weights, i.e., $\Lambda^{+}=\bigoplus_{i=1}^{n} \mathbb{Z}_{\geq 0} \omega_{i}$, where $\omega_{i}=\epsilon_{1}+\cdots+\epsilon_{i}$. There is an alternative description of the affine Weyl group $W$ as the semidirect product

$$
W=W_{0} \ltimes \tau(\Lambda),
$$

where $\tau(\lambda), \lambda \in \Lambda$, is the translation operator given by $\tau(\lambda) f=f+\langle\lambda, f\rangle \delta$ for $f \in \widehat{V}$. The translation operator $\tau\left(\epsilon_{i}\right), i \in[1, n]$, can be expressed in terms of the simple reflections by

$$
\tau\left(\epsilon_{i}\right)=s_{i} \cdots s_{n-1} s_{n} s_{n-1} \cdots s_{1} s_{0} s_{1} \cdots s_{i-1} .
$$

This is a reduced expression.

\subsection{A degenerate affine Hecke algebra}

Let $V_{\mathbb{C}}=V \oplus \mathrm{i} V$ be the complexification of $V$. The finite Weyl group $W_{0}$ acts on $V_{\mathbb{C}}$ by reflections as defined in the previous subsection. We get a representation of the affine Weyl group $W$ on $V_{\mathbb{C}}$ by defining $\tau(\lambda) x=x-\lambda$ for $\lambda \in \Lambda$ and $x \in V_{\mathbb{C}}$. Let $\mathcal{P}$ be the algebra consisting of polynomials on $V_{\mathbb{C}}$, then $W$ acts on $\mathcal{P}$ by $(w p)(x)=p\left(w^{-1} x\right)$, in particular

$$
\begin{aligned}
\left(s_{0} p\right)(x) & =p\left(1-x_{1}, x_{2}, \ldots, x_{n}\right), \\
\left(s_{i} p\right)(x) & =p\left(x_{1}, \ldots, x_{i-1}, x_{i+1}, x_{i}, x_{i+2}, \ldots, x_{n}\right), \quad i \in[1, n-1], \\
\left(s_{n} p\right)(x) & =p\left(x_{1}, \ldots, x_{n-1},-x_{n}\right),
\end{aligned}
$$

where $x=\left(x_{1}, \ldots, x_{n}\right)$. We denote by $\mathcal{P}^{W_{0}}$ the algebra consisting of polynomials $p \in \mathcal{P}$ that are invariant under the action of the finite Weyl group $W_{0}$, i.e., $s_{i} p=p$ for $i \in[1, n]$. The set of monomials $\left\{x^{\mu}\right\}_{\mu \in \mathbb{Z}_{\geq 0}^{n}}$ forms a linear basis for $\mathcal{P}$. Here we use the notation $x^{\mu}=x_{1}^{\mu_{1}} x_{2}^{\mu_{2}} \cdots x_{n}^{\mu_{n}}$ for $x=\left(x_{1}, \ldots, x_{n}\right)$ and $\mu_{i}=\left\langle\mu, \epsilon_{i}\right\rangle$. It will be convenient to label this basis with elements in $\Lambda$. For this we define a bijection $\phi: \mathbb{Z} \rightarrow \mathbb{Z}_{\geq 0}$ by

$$
\phi(m)= \begin{cases}2 m, & m \geq 0, \\ -2 m-1, & m<0 .\end{cases}
$$

Now for $\lambda=\sum \lambda_{i} \epsilon_{i} \in \Lambda$ we denote

$$
\phi(\lambda)=\sum_{i=1}^{n} \phi\left(\lambda_{i}\right) \epsilon_{i} .
$$

Then the set $\left\{x^{\phi(\lambda)}\right\}_{\lambda \in \Lambda}$ is a linear basis for $\mathcal{P}$.

Let $\mathbf{k}: \mathcal{R}_{r} \rightarrow \mathbb{C}$ be a multiplicity function on $\mathcal{R}_{r}$, i.e., $\mathbf{k}$ is constant on $W$-orbits in $\mathcal{R}_{r}$. The function $\mathbf{k}$ is uniquely determined by its values on $a_{0}, a_{1}$ and $a_{n}$, so we may identify $\mathbf{k}$ with the ordered 3 -tuple $\left(k_{0}, k_{1}, k_{n}\right)$, where $k_{i}=\mathbf{k}\left(a_{i}\right)$. Let $\mathcal{P}_{X}$ be the algebra $\mathbb{C}\left[X_{1}, \ldots, X_{n}\right]$. If $p(x)=\sum_{\mu} c_{\mu} x^{\mu} \in \mathcal{P}$ is a polynomial, we denote by $p(X)$ the element $\sum_{\mu} c_{\mu} X^{\mu}$ in $\mathcal{P}_{X}$, where $X^{\mu}=X_{1}^{\mu_{1}} \cdots X_{n}^{\mu_{n}}$ for $\mu_{i}=\left\langle\mu, \epsilon_{i}\right\rangle$. The degenerate (trigonometric) DAHA 
$\mathfrak{H}(\mathbf{k})$ associated to the root system $\mathcal{R}_{r}$ is the complex associative algebra generated by $W$ (with generators $r_{i}, i=0, \ldots, n$ ) and $\mathcal{P}_{X}$, with the cross-relations

$$
r_{i} p(X)-\left(s_{i} p\right)(X) r_{i}=\frac{k_{i}}{a_{i}(X)}\left(\left(s_{i} p\right)(X)-p(X)\right), \quad i \in[0, n] .
$$

Here we denote the generators of $W$ considered as elements of $\mathfrak{H}(\mathbf{k})$ by $r_{i}$ to distinguish them from the operators $s_{i}$. If we say that an algebra $A$ is generated by the algebras $A^{\prime}$ and $A^{\prime \prime}$ (both having a unit element) we mean the following:

- $A \simeq A^{\prime} \otimes A^{\prime \prime}$ as vector spaces,

- for $a^{\prime} \in A^{\prime}$ and $a^{\prime \prime} \in A^{\prime \prime}$ the maps $a^{\prime} \mapsto a^{\prime} \otimes 1$ and $a^{\prime \prime} \mapsto 1 \otimes a^{\prime \prime}$ are algebra homomorphisms,

- $\left(a^{\prime} \otimes 1\right)\left(1 \otimes a^{\prime \prime}\right)=a^{\prime} \otimes a^{\prime \prime}$, for $a^{\prime} \in A^{\prime}, a^{\prime \prime} \in A^{\prime \prime}$.

Moreover, we will write $a^{\prime} a^{\prime \prime}$ instead of $a^{\prime} \otimes a^{\prime \prime}$.

The algebra $\mathfrak{H}(\mathbf{k})$ has a linear basis, called the Poincaré-Birkhoff-Witt (PBW) basis,

$$
\left\{w X^{\phi(\lambda)} \mid w \in W, \lambda \in \Lambda\right\}
$$

Let $\mathbf{t}: \mathcal{R} \rightarrow \mathbb{C}$ be a multiplicity function on $\mathcal{R}$, that we identify with the ordered 5-tuple $\left(\mathbf{t}\left(a_{0}\right), \mathbf{t}\left(a_{0}^{\vee}\right), \mathbf{t}\left(a_{1}\right), \mathbf{t}\left(a_{n}\right), \mathbf{t}\left(a_{n}^{\vee}\right)\right)$. To simplify notation, we write

$$
\mathbf{t}=\left(t_{0}, u_{0}, t, t_{n}, u_{n}\right),
$$

and we also use the notations

$$
\mathbf{t}_{\mathcal{R}_{r}}=\left(t_{0}, t, t_{n}\right) \quad \text { and } \quad \mathbf{t}_{\mathcal{R}_{r}^{\vee}}=\left(u_{0}, t, u_{n}\right) .
$$

We assume that the function $\mathbf{t}$ is related to the multiplicity function $\mathbf{k}$ by

$$
k_{0}=2 t_{0}+2 u_{0}, \quad k_{1}=t, \quad k_{n}=2 t_{n}+2 u_{n} .
$$

We now define a subalgebra $H\left(\mathbf{t}_{\mathcal{R}_{r}}\right)$ of $\mathfrak{H}(\mathbf{k})$ that may be considered as a rational version of the affine Hecke algebra of type $\widetilde{C}_{n}$.

Definition 2.1. The algebra $H=H\left(\mathbf{t}_{\mathcal{R}_{r}}\right) \subset \mathfrak{H}(\mathbf{k})$ is the subalgebra generated by $r_{1}, \ldots, r_{n-1}$ and

$$
T_{i}=t_{i}+\frac{1}{2}\left(t_{i}-u_{i}+a_{i}^{\vee}(X)\right)\left(r_{i}-1\right), \quad i=0, n .
$$

Although it is not clear at this point, the algebra $H$ is an $\left(u_{0}, u_{n}\right)$-dependent subalgebra of $\mathfrak{H}(\mathbf{k})$ that depends as an abstract algebra only on $\mathbf{t}_{\mathcal{R}_{r}}=$ $\left(t_{0}, t, t_{n}\right)$. When working in the algebra $H$ we will write $T_{i}=r_{i}$ for $i \in[1, n-1]$, so $H$ is the algebra generated by $T_{i}, i \in[0, n]$. We also define the algebra $H_{0} \subset H$ to be the subalgebra generated by $T_{i}, i \in[1, n]$. This is the analogue of the finite Hecke algebra of type $C_{n}$. 
Proposition 2.2. We have the following relations in the algebra $H$ :

Quadratic relations:

$$
\begin{aligned}
& T_{i}^{2}=t_{i}^{2}, \quad i=0, n, \\
& T_{i}^{2}=1, \quad i \in[1, n-1],
\end{aligned}
$$

Braid-type relations:

$$
\begin{gathered}
T_{i} T_{i+1} T_{i} T_{i+1}+t T_{i} T_{i+1}=T_{i+1} T_{i} T_{i+1} T_{i}+t T_{i+1} T_{i}, \quad i=0, n-1 \\
T_{i} T_{i+1} T_{i}=T_{i+1} T_{i} T_{i+1}, \quad i \in[1, n-2] \\
T_{i} T_{j}=T_{j} T_{i}, \quad i, j \in[0, n],|i-j| \geq 2 .
\end{gathered}
$$

Proof. We only need to verify the relations involving $T_{0}$ and $T_{n}$, the other ones follow directly from the definition of the algebra $H$. For the quadratic relations we find from the definition of $T_{i}, i=0, n$, and the relations in $\mathfrak{H}(\mathbf{k})$,

$$
\begin{aligned}
& {\left[r_{i}-1\right]\left[t_{i}-u_{i}+a_{i}^{\vee}(X)\right]\left[r_{i}-1\right]} \\
& \quad=\left[\left(t_{i}-u_{i}-a_{i}^{\vee}(X)\right) r_{i}-3 t_{i}-u_{i}-a_{i}^{\vee}(X)\right]\left[r_{i}-1\right] \\
& \quad=-4 t_{i}\left(r_{i}-1\right)
\end{aligned}
$$

which gives

$$
\begin{aligned}
T_{i}^{2}= & t_{i}^{2}+t_{i}\left[t_{i}-u_{i}+a_{i}^{\vee}(X)\right]\left[r_{i}-1\right] \\
& +\frac{1}{4}\left[t_{i}-u_{i}+a_{i}^{\vee}(X)\right]\left(\left[r_{i}-1\right]\left[t_{i}-u_{i}+a_{i}^{\vee}(X)\right]\left[r_{i}-1\right]\right) \\
= & t_{i}^{2} .
\end{aligned}
$$

Next we check the braid-type relation for $T_{n}$. The braid-type relation for $T_{0}$ is checked in the same way. Let us write

$$
f(y)=\frac{1}{2}\left(t_{n}-u_{n}-y\right), \quad g(y)=\frac{1}{2}\left(t_{n}+u_{n}+y\right),
$$

then it follows from the relations in $\mathfrak{H}(\mathbf{k})$ that

$$
T_{n}=t_{n}+\left(s_{n} f\right)\left(X_{n}\right)\left(r_{n}-1\right)=r_{n} f\left(X_{n}\right)-g\left(X_{n}\right) .
$$

Note that we also have

$$
\begin{aligned}
& f\left(X_{n}\right) r_{n-1}=r_{n-1} f\left(X_{n-1}\right)-t / 2, \\
& g\left(X_{n}\right) r_{n-1}=r_{n-1} g\left(X_{n-1}\right)+t / 2 .
\end{aligned}
$$

Now we find in $\mathfrak{H}(\mathbf{k})$

$$
T_{n} r_{n-1}=r_{n} r_{n-1} f\left(X_{n-1}\right)-\frac{1}{2} t r_{n}-r_{n-1} g\left(X_{n-1}\right)-t / 2 .
$$

Multiplying this from the right by $T_{n}=r_{n} f\left(X_{n}\right)-g\left(X_{n}\right)$ we obtain, after some calculations

$$
\begin{aligned}
T_{n} r_{n-1} T_{n}+t T_{n}= & r_{n} r_{n-1} r_{n} f\left(X_{n-1}\right) f\left(X_{n}\right)-r_{n} r_{n-1} f\left(X_{n-1}\right) g\left(X_{n}\right) \\
& -r_{n-1} r_{n} g\left(X_{n-1}\right) f\left(X_{n}\right)+r_{n-1} g\left(X_{n-1}\right) g\left(X_{n}\right) \\
& +\frac{1}{2} t\left(r_{n}-1\right)\left[f\left(X_{n}\right)+g\left(X_{n}\right)\right] .
\end{aligned}
$$


Note that $f(y)+g(y)=t_{n}$. Now we multiply (2.4) from the right by $r_{n-1}$ and bring $r_{n-1}$ to the left of $f$ and $g$, then we have after a few calculations

$$
\begin{aligned}
& T_{n} r_{n-1} T_{n} r_{n-1}+t T_{n} r_{n-1} \\
& \quad=r_{n} r_{n-1} r_{n} r_{n-1} f\left(X_{n}\right) f\left(X_{n-1}\right)-r_{n-1} r_{n} r_{n-1} g\left(X_{n}\right) f\left(X_{n-1}\right) \\
& \quad+\frac{1}{2} t_{n} t r_{n-1} r_{n}-r_{n} f\left(X_{n}\right) g\left(X_{n-1}\right)-\frac{1}{2} t_{n} t r_{n-1}+g\left(X_{n}\right) g\left(X_{n-1}\right) .
\end{aligned}
$$

By inspection, using the braid relations for $r_{n}$, this is the same as (2.4) multiplied by $r_{n-1}$ from the left.

The previous proposition shows that $H$ can be considered as a deformation of the group algebra $\mathbb{C}[W]$, with deformed braid-relations for $i=0, n$.

In order to show that the relations from Proposition 2.2 characterize $H$ as an algebra we show that $H$ has a PBW-basis. We introduce the following notation. If $u$ is a word in the $s_{i}$ 's, $u=s_{i_{1}} \cdots s_{i_{r}}$, then we write $T_{u}=T_{i_{1}} \cdots T_{i_{r}}$.

Proposition 2.3 (PBW-property for $H$ ). For every $w \in W$ let $\bar{w}$ be a fixed reduced expression for $w$, then the set $\left\{T_{\bar{w}} \mid w \in W\right\}$ is a linear basis for $H$.

Proof. The set $\left\{T_{\bar{w}} \mid w \in W\right\}$ spans $H$, see [5, Theorem 2.3]. Now suppose that we have a relation $\sum_{u \in W} c_{u} T_{\bar{u}}=0$ in $H$ for some coefficients $c_{u} \in \mathbb{C}$. Using the cross-relations in $\mathfrak{H}(\mathbf{k})$, we may write

$$
T_{\bar{u}}=\sum_{\substack{w \in W \\ w \leq u}} f_{u, w}(X) w,
$$

where $f_{u, w}(X) \in \mathcal{P}_{X}$ and $f_{u, u}$ is nonzero. Here $w \leq u$ is meant with respect to the Bruhat order on $W$. Now we have

$$
\sum_{\substack{u, w \in W \\ w \leq u}} c_{u} f_{u, w}(X) w=0,
$$

which implies, by the PBW-property for $\mathfrak{H}$, that for any $w \in W$

$$
\sum_{u \geq w} c_{u} f_{u, w}(X)=0 .
$$

Let $v$ be a maximal element (in the Bruhat order) in the set $\left\{u \in W \mid c_{u} \neq 0\right\}$, then $c_{v} f_{v, v}(X)=0$ in $\mathcal{P}_{X}$. But since $f_{v, v}$ is a nonzero polynomial, it follows that $c_{v}=0$. We conclude that $c_{u}=0$ for all $u \in W$.

Corollary 2.4. As an algebra $H$ is completely characterized by the relations from Proposition 2.2 .

Proof. Suppose that we have a complex associative algebra $\mathcal{V}$ generated by $V_{i}$, $i=0, \ldots, n$, with the same relations as in Proposition 2.2 (with $T_{i}$ replaced by $V_{i}$ ). Then the assignments $V_{i} \mapsto T_{i}$ extend to a surjective homomorphism $\psi: \mathcal{V} \rightarrow H$. Now let $V \in \mathcal{V}$ satisfy $\psi(V)=0$. By [5, Theorem 2.3] the set $\left\{V_{\bar{w}} \mid w \in W\right\}$ spans $V$. Writing $V=\sum_{w \in W} c_{w} V_{\bar{w}}$ and applying $\psi$ shows that 
$\sum_{w \in W} c_{w} T_{\bar{w}}=0$, hence every $c_{w}$ is equal to zero by Proposition 2.3. This shows that $\psi$ is also injective.

We also have the PBW-property for the finite algebra $H_{0}$.

Corollary 2.5. The set $\left\{T_{\bar{w}} \mid w \in W_{0}\right\}$ is a linear basis for $H_{0}$.

From Proposition 2.2 and Corollary 2.4 it is now clear that $H$ depends as an abstract algebra only on the parameters $t_{0}, t, t_{n}$. Naturally we also have an algebra $H\left(\mathbf{t}_{\mathcal{R}_{r}^{\vee}}\right)$ related to the reduced root system $\mathcal{R}_{r}^{\vee}$ which is obtained from the algebra $H\left(\mathbf{t}_{\mathcal{R}_{r}}\right)$ by replacing $\left(t_{0}, t_{n}\right)$ by $\left(u_{0}, u_{n}\right)$.

\section{The rational double affine Hecke algebra}

We now come to the definition of the algebra $\mathcal{H}(\mathbf{t})$ that may be considered as a rational degeneration of Sahi's double affine Hecke algebra for type $\left(C^{\vee}, C\right)$. This is explained in Remark 4.2. We call this algebra the rational double affine Hecke algebra. For rank 1 this algebra is studied in [8]. Let us remark that $\mathcal{H}$ is not a rational Cherednik algebra as defined in [4]. We show in Sect. 3.4 that the algebra $\mathcal{H}(\mathbf{t})$ is isomorphic to the rational generalized double affine Hecke algebra attached to an affine Dynkin diagram of type $\widetilde{D}_{4}$ as defined by Etingof et al. [3].

Definition 3.1. The algebra $\mathcal{H}=\mathcal{H}(\mathbf{t}) \subset \mathfrak{H}(\mathbf{k})$ is the subalgebra generated by $H\left(\mathbf{t}_{\mathcal{R}_{r}}\right)$ and $\mathcal{P}_{X}$.

From the relations in $\mathfrak{H}(\mathbf{k})$ it follows that in $\mathcal{H}$ we have, for $p \in \mathcal{P}$,

$$
T_{i} p(X)-\left(s_{i} p\right)(X) T_{i}=d_{i}(X ; \mathbf{t})\left[\left(s_{i} p\right)(X)-p(X)\right], \quad i \in[0, n],
$$

where

$$
d_{i}(X ; \mathbf{t})= \begin{cases}\frac{t_{i}^{2}-u_{i}^{2}+a_{i}^{\vee}(X)^{2}}{a_{i}(X)}, & i=0, n, \\ \frac{t}{a_{i}(X)}, & i \in[1, n-1] .\end{cases}
$$

Together with the PBW-Theorem for the algebra $H$ this leads to the PBW-Theorem for $\mathcal{H}$.

Proposition 3.2 (PBW property for $\mathcal{H})$. The sets

$$
\left\{X^{\phi(\lambda)} T_{\bar{w}} \mid \lambda \in \Lambda, w \in W\right\}, \quad\left\{T_{\bar{w}} X^{\phi(\lambda)} \mid \lambda \in \Lambda, w \in W\right\}
$$

form linear bases for $\mathcal{H}$.

Corollary 3.3. The algebra $\mathcal{H}$ is completely characterized as an algebra by the relations for $H$ from Proposition 2.2, the relations $X_{i} X_{j}=X_{j} X_{i}$ for $i, j \in$ $[1, n]$, and the cross relations (3.1).

We will frequently use (3.1) with $p(X)=X_{i}, i \in[1, n]$. In fact, (3.1) can be recovered from these relations. We collect the identities in the following lemma. 
Lemma 3.4. In $\mathcal{H}$ the following relations hold:

$$
\begin{aligned}
\left(X_{1}-\frac{1}{2}-T_{0}\right)^{2} & =u_{0}^{2}, & & \\
\left(X_{n}+T_{n}\right)^{2} & =u_{n}^{2}, & & \\
T_{i} X_{i} T_{i} & =X_{i+1}-t T_{i}, & & i \in[1, n-1], \\
X_{i} T_{j} & =T_{j} X_{i}, & & i \in[1, n], j \in[0, n],|i-j| \geq 2, \\
X_{i} T_{i+1} & =T_{i+1} X_{i}, & & i \in[1, n-1] .
\end{aligned}
$$

We define in $\mathcal{H}$

$$
T_{0}^{\vee}=X_{1}-\frac{1}{2}-T_{0}, \quad T_{n}^{\vee}=-X_{n}-T_{n},
$$

then we have $\left(T_{0}^{\vee}\right)^{2}=u_{0}^{2}$ and $\left(T_{n}^{\vee}\right)^{2}=u_{n}^{2}$. For $i \in[1, n-1]$ we will sometimes denote $T_{i}^{\vee}=T_{i}$. Let us introduce some convenient notations. We write for $i, j \in[1, n], i<j$,

$$
\begin{aligned}
T_{i, j} & =T_{i} T_{i+1} \cdots T_{j-2} T_{j-1} T_{j-2} \cdots T_{i+1} T_{i} \\
& =T_{j-1} T_{j-2} \cdots T_{i+1} T_{i} T_{i+1} \cdots T_{j-2} T_{j-1} .
\end{aligned}
$$

This notation corresponds to the familiar notation in the symmetric group $\mathcal{S}_{n}$ where $s_{i j}$ denotes the transposition $i \leftrightarrow j$. Recall here that the elements $T_{i}$, $i=1, \ldots, n-1$, generate a subalgebra isomorphic to $\mathcal{S}_{n}$. We also define in $\mathcal{H}$

$$
\begin{aligned}
& \Xi_{i, n}=T_{i} T_{i+1} \cdots T_{n-1} T_{n} T_{n-1} \cdots T_{i+1} T_{i}, \\
& \Xi_{i, n}^{\vee}=T_{i} T_{i+1} \cdots T_{n-1} T_{n}^{\vee} T_{n-1} \cdots T_{i+1} T_{i}, \\
& \Xi_{0, i}=T_{i-1} T_{i-2} \cdots T_{1} T_{0} T_{1} \cdots T_{i-2} T_{i-1}, \\
& \Xi_{0, i}^{\vee}=T_{i-1} T_{i-2} \cdots T_{1} T_{0}^{\vee} T_{1} \cdots T_{i-2} T_{i-1},
\end{aligned}
$$

for $i \in[1, n]$.

We give a few useful relations in $\mathcal{H}$ involving $T_{0}^{\vee}$ and $T_{n}^{\vee}$.

Lemma 3.5. The following relations hold in $\mathcal{H}$ :

$$
\begin{array}{rlrl}
T_{0} T_{1} T_{0}^{\vee} T_{1} & =T_{1} T_{0}^{\vee} T_{1} T_{0} & \\
T_{n} T_{n-1} T_{n}^{\vee} T_{n-1} & =T_{n-1} T_{n}^{\vee} T_{n-1} T_{n} & \\
T_{0}^{\vee} X_{i} & =X_{i} T_{0}^{\vee}, \quad i \in[2, n] \\
T_{n}^{\vee} X_{i} & =X_{i} T_{n}^{\vee}, & \\
T_{0} T_{n}^{\vee} & =T_{n}^{\vee} T_{0} & \\
T_{0}^{\vee} T_{n} & =T_{n} T_{0}^{\vee} & \\
T_{0}^{\vee} T_{n}^{\vee} & =T_{n}^{\vee} T_{0}^{\vee} & \\
\frac{1}{2}+T_{0}+T_{0}^{\vee}+\Xi_{1, n} & \left.+\Xi_{1, n}^{\vee}+t \sum_{j=2}^{n} T_{1, j}=0.1\right]
\end{array}
$$


Proof. Relations (3.2c)-(3.2g) are obvious. For (3.2b) we use the relations

$$
T_{n-1} X_{n} T_{n-1}=X_{n-1}+t T_{n-1}, \quad T_{n} X_{n-1}=X_{n-1} T_{n}
$$

from Lemma 3.4 and the braid-type relations for $T_{n}$;

$$
\begin{aligned}
& T_{n} T_{n-1}\left(X_{n}+T_{n}\right) T_{n-1} \\
& \quad=T_{n}\left(X_{n-1}+t T_{n-1}\right)+T_{n-1} T_{n} T_{n-1} T_{n}+t T_{n-1} T_{n}-t T_{n} T_{n-1} \\
& \quad=\left(X_{n-1}+t T_{n-1}\right) T_{n}+T_{n-1} T_{n} T_{n-1} T_{n} \\
& \quad=T_{n-1}\left(X_{n}+T_{n}\right) T_{n-1} T_{n} .
\end{aligned}
$$

Relation (3.2a) follows in the same way. In order to prove (3.2h), we observe that $T_{i} X_{i+1} T_{i}=X_{i}+t T_{i}$ for $i \in[1, n-1]$, so that by backward induction

$$
T_{1} \cdots T_{n-1} X_{n} T_{n-1} \cdots T_{1}=X_{1}+t \sum_{j=2}^{n} T_{1, j} .
$$

Then (3.2h) follows from the identities $T_{0}^{\vee}+T_{0}+\frac{1}{2}=X_{1}$ and $T_{n}+T_{n}^{\vee}=-X_{n}$, see $(2.3)$.

Next we show that $\mathcal{H}$ has a subalgebra isomorphic to the degenerate affine Hecke algebra $H\left(\mathbf{t}_{\mathcal{R}_{r}^{\vee}}\right)$.

Proposition 3.6. The subalgebra of $\mathcal{H}$ generated by $T_{j}^{\vee}, j=0, \ldots, n$, is isomorphic to $H\left(\mathbf{t}_{\mathcal{R}_{r}^{\vee}}\right)$, i.e., it is characterized by the following relations:

Quadratic relations:

$$
\begin{aligned}
& \left(T_{i}^{\vee}\right)^{2}=u_{i}^{2}, \quad i=0, n, \\
& \left(T_{i}^{\vee}\right)^{2}=1, \quad i \in[1, n-1] .
\end{aligned}
$$

Braid-type relations:

$$
\begin{gathered}
T_{i}^{\vee} T_{i+1}^{\vee} T_{i}^{\vee} T_{i+1}^{\vee}+t T_{i}^{\vee} T_{i+1}^{\vee}=T_{i+1}^{\vee} T_{i}^{\vee} T_{i+1}^{\vee} T_{i}^{\vee}+t T_{i+1}^{\vee} T_{j}^{\vee}, \quad i=0, n-1, \\
T_{i}^{\vee} T_{i+1}^{\vee} T_{i}^{\vee}=T_{i+1}^{\vee} T_{i}^{\vee} T_{i+1}^{\vee}, \quad i \in[1, n-2], \\
T_{i}^{\vee} T_{j}^{\vee}=T_{j}^{\vee} T_{i}^{\vee}, \quad i, j \in[0, n], \quad|i-j| \geq 2 .
\end{gathered}
$$

Proof. Let $H^{\vee}$ be the subalgebra generated by $T_{i}^{\vee}, i=0, \ldots, n$. Let us first verify that the relations mentioned in the proposition are satisfied in $H^{\vee}$. We only need to check the braid-type relations involving $T_{0}^{\vee}$ and $T_{n}^{\vee}$. Let us concentrate on the relations for $T_{n}^{\vee}$. We already know from Lemma 3.5 that $T_{i} T_{n}^{\vee}=T_{n}^{\vee} T_{i}$ for $i \in[1, n-2]$. The relation involving $T_{n-1}$ follows from writing out $X_{n} X_{n-1}=X_{n-1} X_{n}$ if we write $X_{n-1}=T_{n-1} X_{n} T_{n-1}+t T_{n-1}$, $X_{n}=-T_{n}-T_{n}^{\vee}$, and we use the braid-type relation for $T_{n}$ and (3.2b). For $T_{0}^{\vee}$ the proof is similar.

In order to show that the $H^{\vee}$ is indeed isomorphic to $H\left(\mathbf{t}_{\mathcal{R}_{r}^{\vee}}\right)$ it is now enough to show that $\left\{T_{\bar{w}}^{\vee} \mid w \in W\right\}$ is a PBW-basis for $H^{\vee}$. This is done in the same way as in Proposition 2.3. 
We now come to the following characterization of the rational DAHA $\mathcal{H}$, which is the analogue of [18, Theorem 3.4].

Theorem 3.7. The algebra $\mathcal{H}$ is isomorphic to the complex associative algebra $\mathcal{V}$ generated by $V_{0}, V_{0}^{\vee}, V_{n}, V_{n}^{\vee}, V_{i}=V_{i}^{\vee}, i=1, \ldots, n-1$, such that

(i) the subalgebra generated by $V_{i}(i=0, \ldots, n)$ is isomorphic to $H\left(\mathbf{t}_{\mathcal{R}_{r}}\right)$,

(ii) the subalgebra generated by $V_{i}^{\vee}(i=0, \ldots, n)$ is isomorphic to $H\left(\mathbf{t}_{\mathcal{R}_{r}^{\vee}}\right)$,

(iii) the following compatibility relations are satisfied:

$$
\begin{aligned}
& {\left[V_{0}^{\vee}, V_{n}\right]=0, \quad\left[V_{n}^{\vee}, V_{0}\right]=0,} \\
& \frac{1}{2}+V_{0}+V_{0}^{\vee}+\Upsilon_{1, n}+\Upsilon_{1, n}^{\vee}+t \sum_{j=2}^{n} V_{1, j}=0,
\end{aligned}
$$

where

$$
\begin{aligned}
V_{1, j} & =V_{1} V_{2} \cdots V_{j-2} V_{j-1} V_{j-2} \cdots V_{2} V_{1}, \quad j \in[2, n], \\
\Upsilon_{1, n} & =V_{1} V_{2} \cdots V_{n-1} V_{n} V_{n-1} \cdots V_{2} V_{1} \\
\Upsilon_{1, n}^{\vee} & =V_{1} V_{2} \cdots V_{n-1} V_{n}^{\vee} V_{n-1} \cdots V_{2} V_{1} .
\end{aligned}
$$

The isomorphism $\varphi: \mathcal{V} \rightarrow \mathcal{H}$ is given on the generators of $\mathcal{V}$ by

$$
\varphi\left(V_{i}\right)=T_{i}, \quad \varphi\left(V_{i}^{\vee}\right)=T_{i}^{\vee},
$$

for $i \in[0, n]$.

We prove the theorem in the next subsection.

\subsection{Proof of Theorem 3.7}

First note that $\varphi$ preserves the defining relations for $\mathcal{V}$, and $\varphi$ maps generators of $\mathcal{V}$ to generators of $\mathcal{H}$, hence $\varphi$ is a surjective algebra homomorphism. In order to prove that $\varphi$ is also injective, we show that we can recover the defining relations for $\mathcal{H}$ in terms of $T_{0}, \ldots, T_{n}, X_{1}, \ldots, X_{n}$, see Corollary 3.3, from the relations in $\mathcal{V}$. Since the elements $V_{i}, i=0, \ldots, n$, generate an algebra isomorphic to $H\left(\mathbf{t}_{\mathcal{R}_{r}}\right)$, we need to find pairwise commuting elements $v_{i} \in \mathcal{V}$, $i=1, \ldots, n$, such that $\varphi\left(v_{i}\right)=X_{i}$, and the following cross-relations holds, see $(3.1)$ :

$$
V_{i} p(v)-\left(s_{i} p\right)(v) V_{i}=d_{i}(v ; \mathbf{t})\left[\left(s_{i} p\right)(v)-p(v)\right], \quad i \in[0, n] .
$$

Here we use the usual notation $p(v)=\sum_{\mu} c_{\mu} v^{\mu}$, if $p(x)$ is the polynomial $\sum_{\mu} c_{\mu} x^{\mu}$, and $v^{\mu}=v_{1}^{\mu_{1}} \cdots v_{n}^{\mu_{n}}$ for $\mu \in \mathbb{Z}_{\geq 0}^{n}$.

Let us first introduce the elements $\bar{v}_{i}$. We define in $\mathcal{V}$

$$
v_{n}=-V_{n}-V_{n}^{\vee},
$$

and we define $v_{i}$ recursively by

$$
v_{i}=V_{i} v_{i+1} V_{i}-t V_{i}, \quad i \in[1, n-1],
$$


then $\varphi\left(v_{i}\right)=X_{i}$ for $i \in[1, n]$. Note that we may write $v_{i}$ explicitly as

$$
v_{i}=-\left(\Upsilon_{i, n}+\Upsilon_{i, n}^{\vee}+t \sum_{j=i+1}^{n} V_{i, j}\right), \quad i \in[1, n]
$$

where

$$
\begin{aligned}
V_{i, j} & =V_{i} V_{i+1} \cdots V_{j-2} V_{j-1} V_{j-2} \cdots V_{i+1} V_{i}, \quad 1 \leq i<j \leq n, \\
\Upsilon_{i, n} & =V_{i} V_{i+1} \cdots V_{n-1} V_{n} V_{n-1} \cdots V_{i+1} V_{i}, \quad i \in[1, n], \\
\Upsilon_{i, n}^{\vee} & =V_{i} V_{i+1} \cdots V_{n-1} V_{n}^{\vee} V_{n-1} \cdots V_{i+1} V_{i}, \quad i \in[1, n] .
\end{aligned}
$$

For $v_{1}$ the compatibility relation (3.3) gives

$$
v_{1}=\frac{1}{2}+V_{0}+V_{0}^{\vee}
$$

so using the recursion relation for $v_{i}$ again we also have

$$
v_{i}=\frac{1}{2}+\Upsilon_{0, i}+\Upsilon_{0, i}^{\vee}+t \sum_{j=2}^{i} V_{1, j}, \quad i \in[1, n],
$$

where

$$
\begin{aligned}
& \Upsilon_{0, i}=V_{i-1} V_{i-2} \cdots V_{1} V_{0} V_{1} \cdots V_{i-2} V_{i-1}, \quad i \in[1, n] \\
& \Upsilon_{0, i}^{\vee}=V_{i-1} V_{i-2} \cdots V_{1} V_{0}^{\vee} V_{1} \cdots V_{i-2} V_{i-1}, \quad i \in[1, n] .
\end{aligned}
$$

We can now show that we have all the relations from Lemma 3.4 in $\mathcal{V}$.

Lemma 3.8. The following relations hold in $\mathcal{V}$ :

$$
\begin{gathered}
\left(v_{1}-\frac{1}{2}-V_{0}\right)^{2}=u_{0}^{2}, \\
\left(v_{n}+V_{n}\right)^{2}=u_{n}^{2}, \\
V_{i} v_{i} V_{i}=v_{i+1}-t V_{i}, \quad i \in[1, n-1], \\
v_{i} V_{j}=V_{j} v_{i}, \quad j \in[0, n], \quad i \in[1, n],|i-j| \geq 2, \\
v_{i} V_{i+1}=V_{i+1} v_{i}, \quad i \in[1, n-1] .
\end{gathered}
$$

Proof. The first three relations follow directly from the definition of $v_{i}$. Fix $i \in[2, n]$. In $\mathcal{V}$ we have

$$
\begin{aligned}
& {\left[U_{1}, U_{2}\right]=0, \quad U_{1} \in\left\{V_{0}^{\vee}, V_{0}, V_{1}, \ldots, V_{i-2}\right\}} \\
& U_{2} \in\left\{V_{i, i+1}, V_{i, i+2}, \ldots, \Upsilon_{i, n}, \Upsilon_{i, n}^{\vee}\right\}
\end{aligned}
$$


These relations are easily verified using $\left[V_{k}, V_{l}\right]=\left[V_{0}^{\vee}, V_{l}\right]=\left[V_{k}, V_{n}^{\vee}\right]=0$ for $k \in[0, j], l \in[i+1, n]$. Now from (3.6) we find for $j \in[0, i-2]$

$$
\begin{aligned}
V_{j} v_{i} & =-\left(V_{j} \Upsilon_{i, n}+V_{j} \Upsilon_{i, n}^{\vee}+t \sum_{k=i+1}^{n} V_{j} V_{i, k}\right) \\
& =-\left(\Upsilon_{i, n} V_{j}+\Upsilon_{i, n}^{\vee} V_{j}+t \sum_{k=i+1}^{n} V_{i, k} V_{j}\right) \\
& =v_{i} V_{j} .
\end{aligned}
$$

Next we fix $i \in[1, n-1]$. Now we use the relations

$$
\begin{array}{ll}
{\left[U_{1}, U_{2}\right]=0,} & U_{1} \in\left\{V_{i+1}, V_{i+2}, \ldots, V_{n}, V_{n}^{\vee}\right\}, \\
U_{2} & \in\left\{\Upsilon_{0, i}, \Upsilon_{0, i}^{\vee}, V_{1,2}, V_{1,3}, \ldots, V_{1, i}\right\},
\end{array}
$$

then by (3.7) we have for $j \in[i+1, n]$

$$
\begin{aligned}
V_{j} v_{i} & =\frac{1}{2} V_{j}+V_{j} \Upsilon_{0, i}+V_{j} \Upsilon_{0, i}^{\vee}+t \sum_{k=2}^{i} V_{j} V_{1, k} \\
& =\frac{1}{2} V_{j}+\Upsilon_{0, i} V_{j}+\Upsilon_{0, i}^{\vee} V_{j}+t \sum_{k=2}^{i} V_{1, k} V_{j} \\
& =v_{i} V_{j} .
\end{aligned}
$$

We still need to show that the subalgebra generated by $v_{i}, i=1, \ldots, n$, is commutative. Once the commutativity of the $v_{i}$ 's is established, the crossrelations (3.4) follow from Lemma 3.8. The following lemma will be useful for proving the commutativity of the $v_{i}$ 's.

Lemma 3.9. We have the following relations in $\mathcal{V}$ :

$$
\begin{aligned}
& v_{i} V_{0}^{\vee}=V_{0}^{\vee} v_{i}, \quad i \in[2, n], \\
& v_{i} V_{n}^{\vee}=V_{n}^{\vee} v_{i}, \quad i \in[1, n-1], \\
& V_{n} V_{n-1} V_{n}^{\vee} V_{n-1}=V_{n-1} V_{n}^{\vee} V_{n-1} V_{n}
\end{aligned}
$$

Proof. For the first two relations see the proof of Lemma 3.8. The last relation follows from writing out $\left[v_{n-1}, V_{n}\right]=0$ in terms of $V_{n-1}, V_{n}$ and $V_{n}^{\vee}$, and using the braid-type relations for $V_{n}$ and $V_{n-1}$.

We are now ready to prove the commutativity of the $v_{i}$ 's, which completes the proof of Theorem 3.7.

Lemma 3.10. For $i, j \in[1, n]$, we have $v_{i} v_{j}=v_{j} v_{i}$. 
Proof. We first show that $v_{n-1} v_{n}=v_{n} v_{n-1}$. By (3.5) and the definition of $v_{n}$ we have

$$
\begin{aligned}
v_{n-1} v_{n} & =V_{n-1} v_{n} V_{n-1} v_{n}-t V_{n-1} v_{n} \\
& =V_{n-1}\left(V_{n}+V_{n}^{\vee}\right) V_{n-1}\left(V_{n}+V_{n}^{\vee}\right)+t V_{n-1} V_{n}+t V_{n-1} V_{n}^{\vee} .
\end{aligned}
$$

By Lemmas 3.8, 3.9 and the braid-type relations for $V_{n}$ and $V_{n}^{\vee}$ we obtain from this

$$
\begin{aligned}
v_{n-1} v_{n} & =\left(V_{n}+V_{n}^{\vee}\right) V_{n-1}\left(V_{n}+V_{n}^{\vee}\right) V_{n-1}+t V_{n} V_{n-1}+t V_{n}^{\vee} V_{n-1} \\
& =v_{n} V_{n-1} v_{n} V_{n-1}-t v_{n} V_{n-1} \\
& =v_{n} v_{n-1} .
\end{aligned}
$$

Next, we show that $v_{n} v_{i}=v_{i} v_{n}$ by backward induction on $i$. The statement is true for $i=n-1$. Suppose that $v_{n} v_{i+1}=v_{i+1} v_{n}$ for some $i \in[1, n-2]$, then it follows from (3.5) and Lemma 3.8 that

$$
\begin{aligned}
v_{n} v_{i} & =v_{n} V_{i} v_{i+1} V_{i}-t v_{n} V_{i} \\
& =V_{i} v_{n} v_{i+1} V_{i}-t V_{i} v_{n} \\
& =V_{i} v_{i+1} v_{n} V_{i}-t V_{i} v_{n} \\
& =V_{i} v_{i+1} V_{i} v_{n}-t V_{i} v_{n} \\
& =v_{i} v_{n} .
\end{aligned}
$$

This proves the induction step.

Finally, let $i \in[1, n-1]$. A similar induction argument as above shows that $v_{i} v_{j}=v_{j} v_{i}$ for all $j \in[i+1, n]$.

\subsection{The duality isomorphism}

Recall from (2.2) that we identified the multiplicity function $\mathbf{t}$ with the ordered 5 -tuple $\left(t_{0}, u_{0}, t, t_{n}, u_{n}\right)$. We define an involution $\sigma$ on the multiplicity function t by

$$
\mathbf{t}^{\sigma}=\left(u_{n}, u_{0}, t, t_{n}, t_{0}\right)
$$

i.e., the values of $\mathbf{t}$ on the $a_{0}$-orbit and the $a_{n}^{\vee}$-orbit are interchanged. If an object depends on $\mathbf{t}$ we attach a superscript or subscript $\sigma$ to denote the same object depending on $\mathbf{t}^{\sigma}$, for example, $\mathcal{H}_{\sigma}=\mathcal{H}\left(\mathbf{t}^{\sigma}\right)$ and we denote the elements in this algebra by $Z^{\sigma}(Z \in \mathcal{H})$.

For ease of notations, we will write

$$
U_{0}=T_{0}^{\vee}, \quad U_{n}=\Xi_{1, n}^{\vee} .
$$

Note that $U_{i}^{2}=u_{i}^{2}$, and $U_{0}, U_{1}, T_{i}, i \in[0, n]$, form a set of generators for the algebra $\mathcal{H}$.

Proposition 3.11. The assignments

$$
U_{0} \mapsto U_{0}^{\sigma}, \quad U_{n} \mapsto T_{0}^{\sigma}, \quad T_{0} \mapsto U_{n}^{\sigma}, \quad T_{i} \mapsto T_{i}^{\sigma}(i \in[1, n]),
$$

extend uniquely to an isomorphism $\sigma: \mathcal{H} \rightarrow \mathcal{H}_{\sigma}$, respectively an anti-isomorphism $\psi: \mathcal{H} \rightarrow \mathcal{H}_{\sigma}$. 
To prove Proposition 3.11 we only need to check that $\sigma$ and $\psi$ preserve the defining relations for $\mathcal{H}$, which is a straightforward exercise. The only relation that is not obvious is the braid-type relation for $U_{n}$ and $T_{1}$;

$$
U_{n} T_{1} U_{n} T_{1}+t U_{n} T_{1}=T_{1} U_{n} T_{1} U_{n}+t T_{1} U_{n} .
$$

We prove a slightly more general result which is useful later on. The relation in the following lemma with $U=\Xi_{1, n}^{\vee}$ and $j=2$ is the desired braid-type relation for $U_{n}$ and $T_{1}$.

Lemma 3.12. For $j \in[2, n]$ the following braid-type relations hold in $\mathcal{H}$ :

$$
U T_{1, j} U T_{1, j}+t U T_{1, j}=T_{1, j} U T_{1, j} U+t T_{1, j} U, \quad U \in\left\{T_{0}, T_{0}^{\vee}, \Xi_{1, n}, \Xi_{1, n}^{\vee}\right\} .
$$

Proof. Let us first prove the identity for $U=\Xi_{1, n}$. The identity for $U=\Xi_{1, n}^{\vee}$ is proved in exactly the same way. Using $T_{n}=T_{1, n} \Xi_{1, n} T_{1, n}$ and $T_{1, n} T_{1, j} T_{1, n}=$ $T_{j, n}$ we find

$$
\Xi_{1, n} T_{1, j} \Xi_{1, n} T_{1, j}+t \Xi_{1, n} T_{1, j}=T_{1, n}\left(T_{n} T_{j, n} T_{n} T_{j, n}+t T_{n} T_{j, n}\right) T_{1, n} .
$$

We write out $T_{j, n}$ in terms of $T_{i}$ 's, then by repeated application of $\left[T_{i}, T_{j}\right]=0$ and $T_{i}^{2}=1$ the expression between brackets becomes

$$
\begin{aligned}
& T_{n}\left(T_{j} \cdots T_{n-1} \cdots T_{j}\right) T_{n}\left(T_{j} \cdots T_{n-1} \cdots T_{j}\right)+t T_{n}\left(T_{j} \cdots T_{n-1} \cdots T_{j}\right) \\
& \quad=T_{j} \cdots T_{n-2}\left(T_{n} T_{n-1} T_{n} T_{n-1}+t T_{n} T_{n-1}\right) T_{n-2} \cdots T_{j} .
\end{aligned}
$$

Now use the braid-type relations between $T_{n}$ and $T_{n-1}$ and reverse the above steps, then we obtain the desired identity.

For $U=T_{0}, T_{0}^{\vee}$ the desired identities for $j=2$ are precisely the braidtype relation between $U$ and $T_{1}$. For $j \in[3, n]$ the desired identity follow easily from writing $T_{1, j}=T_{j-1} \cdots T_{1} \cdots T_{j-1}$, the braid-type relations between $U$ and $T_{1}$, and repeated application of the relations $\left[U, T_{i}\right]=0$ and $T_{i}^{2}=1$ for $i \in[2, j-1]$.

\subsection{The commutative subalgebra $\mathcal{P}_{Y}$}

For $i \in[1, n]$ we define elements $Y_{i} \in H$ by

$$
Y_{i}=\Xi_{i, n}+\Xi_{0, i}+t \sum_{j=i+1}^{n} T_{i, j} .
$$

From this definition it is clear that

$$
Y_{n}=\Xi_{n, n}+\Xi_{0, n}=T_{n}+T_{n-1} \cdots T_{1} T_{0} T_{1} \cdots T_{n-1},
$$

and

$$
Y_{i+1}=T_{i} Y_{i} T_{i}-t T_{i} .
$$

We denote by $\mathcal{P}_{Y} \subset H \subset \mathcal{H}$ the subalgebra generated by $Y_{1}, \ldots, Y_{n}$. From the definitions of $Y_{i}$ and the algebra $H=H\left(\mathbf{t}_{\mathcal{R}}\right)$ it follows that $H$ is generated as an algebra by $Y_{i}, T_{i}, i=1, \ldots, n$. Observe that $\sigma\left(X_{i}\right)=-Y_{i}^{\sigma}$, so using the duality isomorphism $\sigma$ it is easy to find properties for the $Y_{i}$ 's from the 
properties of the $X_{i}$ 's. Note that we also have $\psi\left(X_{i}\right)=-Y_{i}^{\sigma}$, where $\psi$ is the duality anti-isomorphism.

Proposition 3.13. $\mathcal{P}_{Y}=\mathbb{C}\left[Y_{1}, \ldots, Y_{n}\right]$

Proof. This follows from $\mathcal{P}_{Y}=\sigma\left(\mathcal{P}_{X}^{\sigma}\right), \mathcal{P}_{X}^{\sigma}=\mathbb{C}\left[X_{1}^{\sigma}, \ldots, X_{n}^{\sigma}\right]$, and the fact that $\sigma$ is an isomorphism.

Proposition 3.14. We have the following relations in $H$,

$$
T_{i} p(Y)-\left(s_{i} p\right)(Y) T_{i}=d_{i}\left(Y ; \mathbf{t}^{\sigma}\right)\left[p(Y)-\left(s_{i} p\right)(Y)\right], \quad i \in[1, n],
$$

for $p \in \mathcal{P}$.

Proof. This follows from applying the isomorphism $\sigma_{\sigma}$ to the relations (3.1) in $\mathcal{H}_{\sigma}$.

Corollary 3.15. As a vector space, $H$ is isomorphic to $\mathcal{P}_{Y} \otimes H_{0}$ and $H_{0} \otimes \mathcal{P}_{Y}$. Consequently,

$$
\mathcal{H} \simeq \mathcal{P}_{Y} \otimes H_{0} \otimes \mathcal{P}_{X} \simeq \mathcal{P}_{X} \otimes H_{0} \otimes \mathcal{P}_{Y}
$$

as vector spaces.

Remark 3.16. It should be observed that the elements $Y_{i}$ are not invertible, which is different from the usual (double) affine Hecke algebra.

\subsection{The rational GDAHA for type $\widetilde{D}_{4}$}

We end this section by showing that the rational DAHA $\mathcal{H}(\mathbf{t})$ is isomorphic to the rational generalized double affine Hecke algebra (GDAHA) of rank $n$ attached to an affine Dynkin diagram of type $\tilde{D}_{4}$ as defined in [3, Definition 2.2.1]. We recall the definition here. Actually, in [3] a rational GDAHA of rank $n$ is associated to any star-like graph that is not a finite Dynkin diagram.

Let $\nu \in \mathbb{C}, \mu=\left(\mu_{i_{0}}, \mu_{i_{1}}, \mu_{i_{2}}, \mu_{i_{3}}, \mu_{i_{4}}\right) \in \mathbb{C}^{5}$, and let $\gamma_{k}=\gamma_{k}(\mu)(k \in[1,4])$ be complex numbers such that $\sum_{k=1}^{4} \gamma_{k}=\mu_{i_{0}}$.

Definition 3.17. The algebra $B=B(\mu, \nu)$ is the complex associative algebra generated by $V_{i, k}(i=1, \ldots, n, k=1, \ldots, 4)$ and the symmetric group $\mathcal{S}_{n}$ with the following relations: for any $i, j, k \in[1, n]$ with $i \neq j$, and $l, m \in[1,4]$,

$$
\begin{gathered}
s_{i j} V_{i, l}=V_{j, l} s_{i j}, \\
s_{i j} V_{k, l}=V_{k, l} s_{i j} \quad \text { if } k \neq i, j, \\
\left(V_{k, l}-\gamma_{l}\right)\left(V_{k, l}-\gamma_{l}-\mu_{i_{r}}\right)=0, \\
V_{i, 1}+V_{i, 2}+V_{i, 3}+V_{i, 4}=\nu \sum_{j \neq i} s_{i j}, \\
{\left[V_{i, l}, V_{j, l}\right]=\nu\left(V_{i, l}-V_{j, l}\right) s_{i j},} \\
{\left[V_{i, l}, V_{j, m}\right]=0, \quad l \neq m,}
\end{gathered}
$$

where $s_{i j} \in \mathcal{S}_{n}$ denotes the transposition $i \leftrightarrow j$. 
The algebra $B$ essentially only depends on the parameters $\mu$ and $\nu$, see also [3, Remark 2.2.2]. We are going to show that $B$ is isomorphic to $\mathcal{H}$ for appropriate $\mu$ and $\nu$. For this the following lemma is useful.

Lemma 3.18. The algebra $B$ is generated by $V_{l}:=V_{1, l}(l \in[1,4])$ and $s_{i}:=$ $s_{i, i+1}(i \in[1, n-1])$, with relations, for $l \in[1,4], i \in[1, n-1]$ and $j \in[2, n]$,

$$
\begin{gathered}
s_{i}^{2}=1, \\
s_{i} s_{i+1} s_{i}=s_{i+1} s_{i} s_{i+1}, \\
s_{i} V_{l}=V_{l} s_{i}, \quad i \neq 1, \\
\left(V_{l}-\gamma_{l}\right)\left(V_{l}-\gamma_{l}-\mu_{i_{r}}\right)=0, \\
V_{1}+V_{2}+V_{3}+V_{4}=\nu \sum_{k=2}^{n-1} s_{1 k}, \\
{\left[V_{l}, s_{1 j} V_{l} s_{1 j}\right]=\nu\left[V_{l}, s_{1 j}\right],} \\
{\left[V_{l}, s_{1 j} V_{m} s_{1 j}\right]=0, \quad m \in[1,4], m \neq l,}
\end{gathered}
$$

where $s_{1 j}=s_{1} s_{2} \cdots s_{j-2} s_{j-1} s_{j-2} \cdots s_{2} s_{1}$.

The proof is a straightforward exercise. We can now show that the rational GDAHA $B(\mu, \nu)$, for suitable parameters $\mu$ and $\nu$, is isomorphic to the algebra $\mathcal{H}(\mathbf{t})$, with multiplicity function $\mathbf{t}$ given by $(2.2)$.

Theorem 3.19. Let $\mu(\mathbf{t})=\left(\frac{1}{2}-t_{0}-u_{0}-t_{n}-u_{n}, 2 t_{0}, 2 u_{0}, 2 t_{n}, 2 u_{n}\right)$. The assignments

$$
\begin{aligned}
\varphi\left(s_{i}\right) & =T_{i}, \quad i \in[1, n-1], \\
\varphi\left(V_{1}\right) & =T_{0}+\gamma_{1}+t_{0}, \quad \varphi\left(V_{2}\right)=T_{0}^{\vee}+\gamma_{2}+u_{0}, \\
\varphi\left(V_{3}\right) & =\Xi_{1, n}+\gamma_{3}+t_{n}, \quad \varphi\left(V_{4}\right)=\Xi_{1, n}^{\vee}+\gamma_{4}+u_{n},
\end{aligned}
$$

extend uniquely to an algebra isomorphism $\varphi: B(\mu(\mathbf{t}),-t) \rightarrow \mathcal{H}(\mathbf{t})$.

Proof. Note that $\mathcal{H}$ is generated as an algebra by the elements $T_{0}, T_{0}^{\vee}, \Xi_{1, n}, \Xi_{1, n}^{\vee}$ and $T_{1}, \ldots, T_{n-1}$, hence $\varphi$ maps generators to generators and is therefore surjective. It is an almost straightforward computation to check that $\varphi$ preserves the relations for $B$ from Lemma 3.18. Let us remark that the relations

$$
\left[V_{l}, s_{1 j} V_{l} s_{1 j}\right]=\nu\left[V_{l}, s_{1 j}\right], \quad l \in[1,4], j \in[2, n],
$$

are equivalent to the braid-type relations from Lemma 3.12.

We define $\tilde{\varphi}: \mathcal{H}(\mathbf{t}) \rightarrow B(\mu(\mathbf{t}),-t)$ on generators by

$$
\begin{aligned}
& \tilde{\varphi}\left(T_{i}\right)=s_{i}, \quad i \in[1, n-1], \\
& \tilde{\varphi}\left(T_{0}\right)=V_{1}-\gamma_{1}-t_{0}, \quad \tilde{\varphi}\left(T_{0}^{\vee}\right)=V_{2}-\gamma_{2}-u_{0}, \\
& \tilde{\varphi}\left(T_{n}\right)=s_{1 n} V_{3} s_{1 n}-\gamma_{3}-t_{n}, \quad \tilde{\varphi}\left(T_{n}^{\vee}\right)=s_{1 n} V_{1} s_{1 n}-\gamma_{4}-t_{n} .
\end{aligned}
$$


It is now easily checked that $\tilde{\varphi}$ preserves the relations for $\mathcal{H}$ from Theorem 3.7, so that $\tilde{\varphi}$, extended to $\mathcal{H}$ as an algebra homomorphism, is the inverse of $\varphi$.

\section{The polynomial representation}

In this section we define and study a representation of the rational double affine Hecke algebra $\mathcal{H}$, defined in the previous section, in terms of differencereflection operators.

Recall the algebra $H=H\left(\mathbf{t}_{\mathcal{R}_{r}}\right)$ from Definition 2.1, which can be characterized by the relations from Proposition 2.2, see Corollary 2.4. Let $\chi: H \rightarrow \mathbb{C}$ be the trivial representation defined by $\chi\left(T_{i}\right)=\chi_{i}$ with

$$
\chi_{i}= \begin{cases}t_{i}, & i=0, n, \\ 1, & i \in[1, n-1] .\end{cases}
$$

By Proposition 3.2 we have $\mathcal{H} \simeq H \otimes \mathcal{P}$ as vector spaces, where we identified $\mathcal{P}_{X}$ with $\mathcal{P}$. Now we define the representation $\pi: \mathcal{H} \rightarrow \operatorname{End}(\mathcal{P})$ to be the induced representation

$$
\pi=\operatorname{Ind}_{H}^{\mathcal{H}}(\chi) .
$$

We call $\pi$ the polynomial representation of $\mathcal{H}$. From the cross-relations (3.1) we find the action of the generators of $\mathcal{H}$.

Proposition 4.1. Let $p \in \mathcal{P}$. The actions of the generators $T_{i}(i \in[0, n])$ and $X_{i}(i \in[1, n])$ on $\mathcal{P}$ are given by

$$
\begin{array}{rlrl}
\left(\pi\left(T_{i}\right) p\right)(x) & =c_{i}(x)\left(s_{i} p\right)(x)-d_{i}(x) p(x), & & i \in[0, n], \\
\left(\pi\left(X_{i}\right) p\right)(x) & =x_{i} p(x), & i \in[1, n],
\end{array}
$$

where $c_{i}(x)=c_{i}(x ; \mathbf{t})$ are rational functions defined by

$$
c_{i}(x)=d_{i}(x)+\chi_{i}, \quad i \in[0, n] .
$$

Explicitly, the rational functions $c_{i}$ are given by

$$
c_{i}(x)= \begin{cases}\frac{\left(t_{i}+u_{i}+a_{i}^{\vee}(x)\right)\left(t_{i}-u_{i}+a_{i}^{\vee}(x)\right)}{a_{i}(x)}, & i=0, n, \\ \frac{t+a_{i}(x)}{a_{i}(x)}, & i \in[1, n-1] .\end{cases}
$$

An easy computation shows that

$$
c_{i}(x)+\left(s_{i} c_{i}\right)(x)=2 \chi_{i}, \quad i \in[0, n] .
$$

We will also use the notation, for $\alpha \in \mathcal{R}_{r}$,

$$
c_{\alpha}(x ; \mathbf{t})= \begin{cases}\frac{\left(\left(\mathbf{t}(\alpha)+\mathbf{t}\left(\alpha^{\vee}\right)+\alpha^{\vee}(x)\right)\left(\mathbf{t}(\alpha)-\mathbf{t}\left(\alpha^{\vee}\right)+\alpha^{\vee}(x)\right)\right.}{\alpha(x)}, & \langle\alpha, \alpha\rangle=4, \\ \frac{\mathbf{t}(\alpha)+\alpha(x)}{\alpha(x)}, & \langle\alpha, \alpha\rangle=2 .\end{cases}
$$

Recalling that the multiplicity function $\mathbf{t}$ is given by (2.2), we see that $c_{a_{i}}=c_{i}$ for $i \in[0, n]$, and $w c_{\alpha}=c_{w \alpha}$ for $w \in W$. 
Remark 4.2. (i) Note that for $t=0$ we have $\pi\left(T_{i}\right)=s_{i}, i \in[1, n-1]$. Moreover, setting $\left(t_{0}, u_{0}, t_{n}, u_{n}\right)=(a,-a, a,-a)$ we find

$$
\lim _{a \rightarrow \infty}\left(\pi\left(t_{i}^{-1} T_{i}\right) p\right)(x)=\left(s_{i} p\right)(x), \quad i=0, n,
$$

for $p \in \mathcal{P}$.

(ii) Noumi's [14] representation of the affine Hecke algebra of type $\widetilde{C}_{n}$ on $\mathbb{C}\left[q^{ \pm x_{1}}, \ldots, q^{ \pm x_{n}}\right]$ is given by $V_{i}=k_{a_{i}}+k_{a_{i}}^{-1} \frac{\left(1-k_{a_{i}} k_{a_{i} / 2} q^{a_{i}(x) / 2}\right)\left(1+k_{a_{i}} k_{a_{i} / 2}^{-1} q^{a_{i}(x) / 2}\right)}{1-q^{a_{i}(x)}}\left(s_{i}-1\right), \quad i \in[0, n]$. Setting

Here $k$ is a multiplicity function on $\mathcal{R}_{n r}$, and $k_{a_{i} / 2}=1$ if $a_{i} / 2 \notin \mathcal{R}_{n r}$.

$$
\left(k_{a_{0}}, k_{a_{0} / 2}, k_{a_{1}}, k_{a_{n}}, k_{a_{n} / 2}\right)=\left(-\mathrm{i} q^{t_{0}}, \mathrm{i} q^{u_{0}}, q^{t / 2},-\mathrm{i} q^{t_{n}}, \mathrm{i} q^{u_{n}}\right),
$$

we find formally for $q \rightarrow 1$ (see also [8, Section 2.4] and [1, Sect. 4])

$$
\begin{array}{ll}
\lim _{q \rightarrow 1} \frac{1-\mathrm{i} V_{i}}{1-q}=\pi\left(T_{i}\right), & i=0, n, \\
\lim _{q \rightarrow 1} V_{i}=\pi\left(T_{i}\right), & i \in[1, n-1] .
\end{array}
$$

So the representation $\pi$ of $\mathcal{H}$ can formally be obtained as a limit of Noumi's representation of the DAHA associated to $\mathcal{R}_{n r}$, see [16]. In view of the next proposition the algebra $\mathcal{H}$ may be considered as a degeneration of the DAHA associated to $\mathcal{R}_{n r}$.

Proposition 4.3. The representation $\pi: \mathcal{H} \rightarrow \operatorname{End}(\mathcal{P})$ is faithful.

Proof. Suppose that $\pi\left(\sum_{w \in W} f_{w}(X) T_{\bar{w}}\right)=0$, with $f_{w}(X) \in \mathcal{P}_{X}$. We may write $\pi\left(T_{\bar{w}}\right)=\sum_{u \leq w} a_{w, u}(x) u$ with $a_{w, u}(x) \in \mathbb{C}(x)$ and $a_{w, w}(x)$ is nonzero. Let $d(x)$ be the product of the denominators of all $a_{w, u}(x)$, then

$$
0=d(x) \sum_{\substack{u, w \in W \\ u \leq w}} f_{w}(x) a_{w, u}(x) u .
$$

We may consider the expression on the right as an element of the degenerate DAHA $\mathfrak{H}(\mathbf{0}) \simeq \mathcal{P} \otimes W$, with $\mathbf{0}$ the multiplicity function which is equal to zero on all $W$-orbits in $\mathcal{R}_{r}$. Now it follows in the same way as in the proof of Proposition 2.3 that $f_{w}(x)$ is the polynomial identically equal to zero for all $w \in W$, hence $\pi$ is a faithful representation of $\mathcal{H}$.

The representation $\pi$ restricted to the subalgebra $H$ gives a representation of $H$. We are going to decompose $\mathcal{P}$, considered as a $\pi(H)$-module, into irreducible $\pi(H)$-modules. In view of Proposition 4.3 we identify from here on the algebra $\mathcal{H}$ with the algebra $\pi(\mathcal{H}) \subset \operatorname{End}(\mathcal{P})$.

It will be useful to understand the action of the operators $T_{i}$ on monomials. For $\nu=\sum_{i=1}^{n} \nu_{i} \epsilon_{i} \in \mathbb{Z}_{\geq 0}^{n}$ we write $|\nu|=\sum_{i=1}^{n} \nu_{i}$. 
Lemma 4.4. For $\lambda \in \Lambda$ and $\nu=\phi(\lambda)$, we have

$$
\begin{aligned}
& T_{0} x^{\nu}= \begin{cases}\left(t_{0}+\left\langle\lambda, \epsilon_{1}\right\rangle\right) x^{\nu}+\sum_{|\mu|<|\nu|} c_{\nu \mu} x^{\mu}, & \left\langle\lambda, \epsilon_{1}\right\rangle \geq 0, \\
x^{\phi\left(s_{\epsilon_{1}} \lambda\right)}-\left(t_{0}-\left\langle\lambda, \epsilon_{1}\right\rangle\right) x^{\nu}+\sum_{|\mu|<|\nu|} c_{\nu \mu} x^{\mu}, & \left\langle\lambda, \epsilon_{1}\right\rangle<0,\end{cases} \\
& T_{i} x^{\nu}=x^{\phi\left(s_{i} \lambda\right)}+\sum_{|\mu|<|\nu|} c_{\nu \mu} x^{\mu}, \quad i \in[1, n-1], \\
& T_{n} x^{\nu}= \begin{cases}t_{n} x^{\nu}, & \left\langle\lambda, \epsilon_{n}\right\rangle \geq 0, \\
-x^{\phi\left(s_{n} \lambda\right)}-t_{n} x^{\nu}+\sum_{|\mu|<|\nu|} c_{\nu \mu} x^{\mu}, & \left\langle\lambda, \epsilon_{n}\right\rangle<0,\end{cases}
\end{aligned}
$$

for certain coefficients $c_{\nu \mu} \in \mathbb{C}$.

Proof. For $T_{0}$ and $T_{n}$ this is proved in [8, Proposition 2.5]. For $T_{i}, i \in[1, n-1]$, we write $T_{i}=s_{i}+t D_{i}$, where

$$
\left(D_{i} p\right)(x)=\frac{\left(s_{i} p\right)(x)-p(x)}{a_{i}(x)}, \quad p \in \mathcal{P} .
$$

We use $s_{i}\left(x^{\nu}\right)=x^{s_{i} \nu}$. Then from $s_{i} \nu=\nu-m a_{i}$, where $m=\left\langle\nu, a_{i}\right\rangle$, we obtain

$$
\begin{aligned}
D_{i} x^{\nu} & =\frac{\left(s_{i} x\right)^{\nu}-x^{\nu}}{a_{i}(x)}=\frac{x^{\nu}\left(x^{-m a_{i}}-1\right)}{x_{i+1}\left(x^{a_{i}}-1\right)} \\
& = \begin{cases}-x^{\nu-\epsilon_{i+1}-a_{i}}-x^{\nu-\epsilon_{i+1}-2 a_{i}}-\cdots-x^{\nu-\epsilon_{i+1}-m a_{i}}, & m>0, \\
0, & m=0 \\
x^{\nu-\epsilon_{i+1}}+x^{\nu-\epsilon_{i+1}+a_{i}}+\cdots+x^{\nu-\epsilon_{i+1}-(1+m) a_{i}}, & m<0 .\end{cases}
\end{aligned}
$$

All these terms are of degree lower than $|\nu|$.

\subsection{Intertwiners}

For $i \in[0, n]$ we define the elements $S_{i} \in \mathcal{H}$, called intertwiners, by

$$
\begin{aligned}
S_{0} & =\left[U_{n}, a_{0}(Y)\right], \\
S_{i} & =\left[T_{i}, a_{i}(Y)\right], \quad i \in[1, n] .
\end{aligned}
$$

We define another action of $W$ on $V_{\mathbb{C}}$ by $s_{i} \cdot x=s_{i} x$ for $i \in[1, n]$ and

$$
s_{0} \cdot x=\left(-x_{1}-1, x_{2}, \ldots, x_{n}\right), \quad x=\left(x_{1}, \ldots, x_{2}\right) \in V_{\mathbb{C}} .
$$

We also define a dot-action of $W$ on $\mathcal{P}$ by $(w \cdot p)(x)=p\left(w^{-1} \cdot x\right)$. Note in particular that the action of the commutative subalgebra $\tau(\Lambda)$ is given by $\left(\tau\left(\epsilon_{i}\right) \cdot p\right)(x)=p\left(x-\epsilon_{i}\right)$.

Lemma 4.5. The intertwiners $S_{i}$ satisfy the following relations in $\mathcal{H}$ :

(i) The braid relations of type $\widetilde{C}_{n}$

$$
\begin{aligned}
& S_{i} S_{i+1} S_{i} S_{i+1}=S_{i+1} S_{i} S_{i+1} S_{i}, \quad i=0, n-1, \\
& S_{i} S_{i+1} S_{i}=S_{i+1} S_{i} S_{i+1}, \quad i \in[1, n-2], \\
& S_{i} S_{j}=S_{j} S_{i}, \quad i, j \in[0, n], \quad|i-j| \geq 2 .
\end{aligned}
$$


(ii) The quadratic relations $S_{i}^{2}=q_{i}(Y)$ with $q_{i}(x)=-2 a_{i}(x)^{2} c_{a_{i}}(x) c_{-a_{i}}(x)$, i.e.,

$q_{i}(x)= \begin{cases}4\left(\left(u_{n}+u_{0}\right)^{2}-\left(\frac{1}{2}+x_{1}\right)^{2}\right)\left(\left(u_{n}-u_{0}\right)^{2}-\left(\frac{1}{2}+x_{1}\right)^{2}\right), & i=0, \\ 4\left(t^{2}-a_{i}(x)^{2}\right), & i \in[1, n-1], \\ 4\left(\left(t_{n}+t_{0}\right)^{2}-x_{n}^{2}\right)\left(\left(t_{n}-t_{0}\right)^{2}-x_{n}^{2}\right), & i=n .\end{cases}$

(iii) For $p \in \mathcal{P}$,

$$
S_{i} p(Y)=\left(s_{i} \cdot p\right)(Y) S_{i}, \quad i \in[0, n] .
$$

Proof. First we apply the duality isomorphism $\sigma: \mathcal{H} \rightarrow \mathcal{H}_{\sigma}$ and then the representation $\pi_{\sigma}: \mathcal{H}_{\sigma} \rightarrow \operatorname{End}(\mathcal{P})$, then by (3.1) we have

$$
\left(\pi_{\sigma} \circ \sigma\right)\left(S_{i}\right)=-2 a_{i}(x) c_{i}(x) s_{i} .
$$

Properties (i) and (ii) follow from this after an easy calculation. Property (iii) follows in the same way, using also $\left(\pi_{\sigma} \circ \sigma\right)(p(Y))=p(-x)$.

For $w \in W$ we define intertwiners $S_{w}$ by $S_{1}=1$, and

$$
S_{w}=S_{i_{1}} \cdots S_{i_{r}}
$$

for $w=s_{i_{1}} \cdots s_{i_{r}} \in W$ a reduced expression. This is independent of the choice of the reduced expression, since the $S_{i}$ 's satisfy the braid relations of type $\widetilde{C}_{n}$. From Proposition 4.5 we now find the following relation in $\mathcal{H}$.

Corollary 4.6. For $p \in \mathcal{P}$ and $w \in W$ the following relation holds in $\mathcal{H}$ :

$$
S_{w}\left(w^{-1} \cdot p\right)(Y)=p(Y) S_{w} .
$$

\subsection{Nonsymmetric multivariable Wilson polynomials}

For $\lambda \in \Lambda$ let $\lambda^{+}$be the unique element in $W_{0} \lambda \cap \Lambda^{+}$. There is a unique shortest element (with respect to the length-function $l$ on $W$ ) $v_{\lambda} \in W_{0}$ such that $v_{\lambda} \cdot \lambda=\lambda^{+}$. We define $u_{\lambda} \in W$ by $u_{\lambda}=\tau(-\lambda) v_{\lambda}^{-1}$. We give a few useful properties of $u_{\lambda}$, which are proved in [12, Sect. 2.4].

Lemma 4.7. For $\lambda \in \Lambda$ the element $u_{\lambda}$ has the following properties:

(i) $\tau(-\lambda)=u_{\lambda} v_{\lambda}$ and $\tau\left(-\lambda^{+}\right)=v_{\lambda} u_{\lambda}$.

(ii) $u_{\lambda}$ is the unique shortest element in $W$ satisfying $u_{\lambda} \cdot 0=\lambda$.

(iii) If $a_{i}(\lambda) \neq 0$ for $i \in[0, n]$, then $s_{i} u_{\lambda}=u_{s_{i} \cdot \lambda}$.

(iv) If $a_{i}(\lambda)<0$ for $i \in[0, n]$, then $l\left(u_{s_{i} \cdot \lambda}\right)=l\left(u_{\lambda}\right)-1$.

(v) If $a_{i}(\lambda)=0$ for $i \in[1, n]$, then there exists a $j \in[1, n]$ such that $s_{i} u_{\lambda}=$ $u_{\lambda} s_{j}$.

We now define polynomials labeled by $\lambda \in \Lambda$ generated by the intertwiners from the constant polynomial $1 \in \mathcal{P}$, see also [13].

Definition 4.8. For $\lambda \in \Lambda$ we define the nonsymmetric multivariable Wilson polynomial $p_{\lambda} \in \mathcal{P}$ by

$$
p_{\lambda}=S_{u_{\lambda}} 1 \text {. }
$$


We see that $p_{\lambda}$ is a nonzero polynomial for any $\lambda \in \Lambda$. Observe that $p_{\lambda}$ depends on the five parameters from the multiplicity function $\mathbf{t}$, see (2.2). To stress this dependence we sometimes write $p_{\lambda}=p_{\lambda}(\cdot ; \mathbf{t})$. We will show that the polynomials $p_{\lambda}$ are eigenfunctions of the $Y$-operators.

Remark 4.9. We show later on that the polynomials $p_{\lambda}$ are nonsymmetric versions of the multivariable Wilson polynomials as defined by van Diejen [20], which justifies the name. In this paper we will often call $p_{\lambda}$ a Wilson polynomial.

Note that the constant function $p_{0}=1 \in \mathcal{P}$ satisfies $T_{i} p_{0}=\chi_{i} p_{0}$ for $i \in[0, n]$, see (4.1). Now from the definition of the $Y$-operators we see that

$$
Y_{i} p_{0}=\gamma_{0, i} p_{0}, \quad \gamma_{0, i}=t_{0}+t_{n}+(n-i) t .
$$

For $\lambda \in \Lambda$ we define elements $\gamma_{\lambda} \in V_{\mathbb{C}}$ as follows:

$$
\gamma_{\lambda}=u_{\lambda} \cdot \gamma_{0}, \quad \gamma_{0}=\sum_{i=1}^{n} \gamma_{0, i} \epsilon_{i} .
$$

From the definition of $u_{\lambda}$ it follows that $\gamma_{\lambda}$ can also be written as $\lambda+v_{\lambda}^{-1} \cdot \gamma_{0}$. From here on we assume that the parameters $t_{0}, t_{n}, t$ are such that $\gamma_{\lambda} \neq \gamma_{\mu}$ if $\mu \neq \lambda$. We have the following useful lemma.

Lemma 4.10. Let $p \in \mathcal{P}$ and $\lambda \in \Lambda$, then

(i) $s_{i} \cdot \gamma_{\lambda}=\gamma_{s_{i} \cdot \lambda}$ for $i \in[0, n]$.

(ii) $\left(s_{i} p\right)\left(-\gamma_{\lambda}\right)=p\left(-\gamma_{s_{i} \cdot \lambda}\right)$ and $\left(s_{i} \cdot p\right)\left(\gamma_{\lambda}\right)=p\left(\gamma_{s_{i} \cdot \lambda}\right)$ for $i \in[0, n]$ with $s_{i} \cdot \lambda \neq \lambda$.

For the proof see [16, Theorem 5.3].

Theorem 4.11. For $f \in \mathcal{P}$ and $\lambda \in \Lambda$, we have

$$
f(Y) p_{\lambda}=f\left(\gamma_{\lambda}\right) p_{\lambda} .
$$

Proof. By Corollary 4.6 and Lemma 4.10 we have

$$
\begin{aligned}
f(Y) p_{\lambda} & =f(Y) S_{u_{\lambda}} 1=S_{u_{\lambda}}\left(u_{\lambda}^{-1} \cdot f\right)(Y) 1 \\
& =\left(u_{\lambda}^{-1} \cdot f\right)\left(\gamma_{0}\right) S_{u_{\lambda}} 1=f\left(\gamma_{u_{\lambda} \cdot 0}\right) p_{\lambda}=f\left(\gamma_{\lambda}\right) p_{\lambda} .
\end{aligned}
$$

For $\lambda \in \Lambda$ we define eigenspaces

$$
\mathcal{P}_{\lambda}=\left\{p \in \mathcal{P} \mid f(Y) p=f\left(\gamma_{\lambda}\right) p \text { for all } f \in \mathcal{P}\right\} .
$$

Clearly, we have $p_{\lambda} \in \mathcal{P}_{\lambda}$, so $\mathcal{P}_{\lambda}$ is nonempty and nonzero for every $\lambda \in \Lambda$.

Theorem 4.12. The set $\left\{p_{\lambda} \mid \lambda \in \Lambda\right\}$ is a basis for $\mathcal{P}$. 
Proof. We first show that the eigenspaces $\mathcal{P}_{\lambda}$ are all 1-dimensional. Let $p \in \mathcal{P}_{\lambda}$. Observe that for $t=0$ we have $T_{i}=s_{i}, i \in[1, n-1]$, which gives us for the $Y$-operators

$$
Y_{i}=s_{i} \cdots s_{n-1} T_{n} s_{n-1} \cdots s_{i}+s_{i-1} \cdots s_{1} T_{0} s_{1} \cdots s_{i-1} .
$$

This is the $Y$-operator for the rank one version of the algebra $\mathcal{H}$ (see [8]), acting only on the $i$ th variable. For any $m \in \mathbb{Z}_{\geq 0}$ the rank one $Y$-operator has exactly one (up to a multiplicative constant) polynomial eigenfunction of degree $m$, which is uniquely determined by the coefficient of $x^{m}$. This means that for $t=0$ we have

$$
p(x)=c p_{\phi\left(\lambda_{1}\right)}\left(x_{1}\right) p_{\phi\left(\lambda_{2}\right)}\left(x_{2}\right) \cdots p_{\phi\left(\lambda_{n}\right)}\left(x_{n}\right),
$$

where $p_{m}$ denotes a one-variable nonsymmetric Wilson polynomial of degree $m$, and $c$ is a nonzero constant. So the coefficient $c_{\lambda}$ of $x^{\phi(\lambda)}$ in the expansion $p(x)=\sum_{\mu \in \Lambda} c_{\mu} x^{\phi(\mu)}$ is nonzero after setting $t=0$, which implies that $c_{\lambda}$ is generically nonzero. Now if $\operatorname{dim} \mathcal{P}_{\lambda}>1$, there would be a nonzero polynomial in $\mathcal{P}_{\lambda}$ for which the coefficient of $x^{\phi(\lambda)}$ is equal to zero, hence $\operatorname{dim} \mathcal{P}_{\lambda}=1$.

Finally, we define for $m \in \mathbb{Z}_{\geq 0}$,

$$
\mathcal{P}_{(m)}=\operatorname{span}\left\{x^{\phi(\lambda)}\left|\lambda=\sum_{i=1}^{n} \lambda_{i} \epsilon_{i} \in \Lambda, \sum_{i=1}^{n}\right| \lambda_{i} \mid \leq m\right\},
$$

then $\mathcal{P}=\bigcup_{m=0}^{\infty} \mathcal{P}_{(m)}$. Since $\sum_{i} \lambda_{i}^{+}=\sum_{i}\left|\lambda_{i}\right|$ for all $\lambda \in \Lambda$, we see from Lemma 4.4 that the intertwiners $S_{i}, i \in[1, n]$, satisfy $S_{i} \mathcal{P}_{(m)} \subset \mathcal{P}_{(m)}$, while $S_{0} \mathcal{P}_{(m)} \subset \mathcal{P}_{(m+1)}$. It follows that $p_{\lambda} \in \mathcal{P}_{(N)}$ where $N$ is the number of times $s_{0}$ occurs in a reduced expression for $u_{\lambda}$. By Lemma 4.7 we have $u_{\lambda}=v_{\lambda}^{-1} \tau\left(-\lambda^{+}\right)$ with $v_{\lambda} \in W_{0}$, so $N$ is also the number of times $s_{0}$ occurs in $\tau\left(-\lambda_{+}\right)$, i.e., $N=\sum_{i} \lambda_{i}^{+}$. So for any $m \in \mathbb{Z}_{\geq 0}$ the set $\left\{p_{\lambda}\left|\sum_{i}\right| \lambda_{i} \mid \leq m\right\} \subset \mathcal{P}_{(m)}$ has the same cardinality as the basis $\left\{x^{\phi(\lambda)}\left|\sum_{i}\right| \lambda_{i} \mid \leq m\right\}$ of $\mathcal{P}_{(m)}$. Since the polynomials $p_{\lambda}, \sum_{i}\left|\lambda_{i}\right| \leq m$, are eigenfunctions of the $Y$-operators for pairwise different eigenvalues, they are linear independent in $\mathcal{P}_{(m)}$, hence the set $\left\{p_{\lambda}\left|\sum_{i}\right| \lambda_{i} \mid \leq m\right\}$ forms a basis for $\mathcal{P}_{(m)}$.

We have the following corollary of the proof of Theorem 4.12.

Corollary 4.13. The eigenspaces $\mathcal{P}_{\lambda}, \lambda \in \Lambda$, are all one-dimensional. Furthermore, a polynomial in $\mathcal{P}_{\lambda}$ is uniquely determined by the coefficient of $x^{\phi(\lambda)}$.

We define for $\lambda \in \Lambda^{+}$,

$$
\mathcal{P}(\lambda)=\operatorname{span}\left\{p_{\mu} \mid \mu \in W_{0} \lambda\right\} .
$$

We are going to show that $\mathcal{P}(\lambda)$ is an irreducible $\pi(H)$-module.

Lemma 4.14. Let $\lambda \in \Lambda$ and $i \in[0, n]$, then

$$
S_{i} p_{\lambda}= \begin{cases}0, & a_{i}(\lambda)=0 \\ p_{s_{i} \cdot \lambda}, & a_{i}(\lambda)>0 \\ q_{i}\left(\gamma_{s_{i} \cdot \lambda}\right) p_{s_{i} \cdot \lambda}, & a_{i}(\lambda)<0\end{cases}
$$


Proof. Let us first assume that $a_{i}(\lambda) \neq 0$, i.e., $s_{i} \cdot \lambda \neq \lambda$. We have $S_{i} p_{\lambda}=$ $S_{i} S_{u_{\lambda}}$ 1. Let $s_{i_{1}} \cdots s_{i_{r}}$ be a reduced expression for $u_{\lambda}$. We need to find out if $s_{i} s_{i_{1}} \cdots s_{i_{r}}$ is a reduced expression for $s_{i} u_{\lambda}$. By Lemma 4.7 we have $u_{s_{i} \cdot \lambda}=s_{i} u_{\lambda}$. If $l\left(u_{s_{i} \cdot \lambda}\right)>l\left(u_{\lambda}\right)$, then $s_{i} s_{i_{1}} \cdots s_{i_{r}}$ is a reduced expression for $u_{s_{i} \cdot \lambda}$. In this case $S_{i} S_{u_{\lambda}}=S_{u_{s_{i} \cdot \lambda}}$, which implies $S_{i} p_{\lambda}=p_{s_{i} \cdot \lambda}$. If $l\left(u_{s_{i} \cdot \lambda}\right)<$ $l\left(u_{\lambda}\right)$, then there exists a reduced expression for $u_{\lambda}$ which starts with $s_{i}$. This shows that $S_{i} S_{u_{\lambda}}=S_{i}^{2} S_{u_{s_{i} \cdot \lambda}}$. From Lemma 4.5 and Theorem 4.11 we then obtain $S_{i} p_{\lambda}=S_{i}^{2} p_{s_{i} \cdot \lambda}=q_{i}\left(\gamma_{s_{i} \cdot \lambda}\right) p_{s_{i} \cdot \lambda}$. By Lemma $4.7 a_{i}(\lambda)<0$ implies $l\left(u_{s_{i} \cdot \lambda}\right)<l\left(u_{\lambda}\right)$. Replacing $\lambda$ by $s_{i} \cdot \lambda$ then shows that $a_{i}(\lambda)>0$ implies $l\left(u_{s_{i} \cdot \lambda}\right)>l\left(u_{\lambda}\right)$. This proves the lemma in case $a_{i}(\lambda) \neq 0$.

Now suppose that $a_{i}(\lambda)=0$, i.e., $s_{i} \cdot \lambda=\lambda$, which only happens if $i \neq 0$. In this case we have $\left(s_{i} u_{\lambda}\right) \cdot 0=s_{i} \cdot \lambda=\lambda$. Since $u_{\lambda}$ is the shortest element in $W$ satisfying $u_{\lambda} \cdot 0=\lambda$, we have $l\left(s_{i} u_{\lambda}\right)>l\left(u_{\lambda}\right)$. Furthermore, by Lemma 4.7 there exists a $j \in[1, n]$ such that $s_{i} u_{\lambda}=u_{\lambda} s_{j}$. This shows that $S_{i} p_{\lambda}=S_{i} S_{u_{\lambda}} 1=S_{u_{\lambda}} S_{j} 1$. Observe that $S_{j} 1=\left[T_{j}, a_{j}(Y)\right] 1=0$, since $T_{j} 1=\chi_{j}$, hence $S_{i} p_{\lambda}=0$.

The following result assures us that $T_{i} \mathcal{P}(\lambda) \subset \mathcal{P}(\lambda)$ for $i \in[1, n]$. Clearly, we also have $Y_{i} \mathcal{P}(\lambda) \subset \mathcal{P}(\lambda)$ for $i \in[1, n]$. Since the $Y$-operators are diagonalized by $p_{\mu}$, a nonempty invariant subspace $\widetilde{\mathcal{P}} \subset \mathcal{P}(\lambda)$ contains an element $p_{\mu}$ for some $\mu \in W_{0} \lambda$. Repeated application of the intertwiners $S_{i}, i \in[1, n]$, then shows that for every $\nu \in W_{0} \lambda$ we have $p_{\nu} \in \widetilde{\mathcal{P}}$, hence $\mathcal{P}(\lambda)$ is irreducible.

Lemma 4.15. Let $\lambda \in \Lambda$ and $i \in[1, n]$. If $a_{i}(\lambda) \neq 0$, then

$$
T_{i} p_{\lambda}=A_{i, \lambda} p_{\lambda}+B_{i, \lambda} p_{s_{i} \cdot \lambda},
$$

with $A_{i, \lambda}=d_{i}^{\sigma}\left(\gamma_{\lambda}\right)$ and

$$
B_{i, \lambda}= \begin{cases}\frac{1}{2 a_{i}\left(\gamma_{\lambda}\right)}, & a_{i}(\lambda)>0, \\ \frac{q_{i}\left(\gamma_{s_{i} \cdot \lambda}\right)}{2 a_{i}\left(\gamma_{\lambda}\right)}, & a_{i}(\lambda)<0 .\end{cases}
$$

If $a_{i}(\lambda)=0$, then $T_{i} p_{\lambda}=\chi_{i} p_{\lambda}$.

Proof. Let $i \in[1, n]$ and $\lambda \in \Lambda$. By Theorem $4.12 T_{i} p_{\lambda}$ can be expanded in terms of $p_{\mu}, \mu \in \Lambda$. From the cross-relation in Proposition 3.14 we find for any $f \in \mathcal{P}$,

$$
\left(f(Y)-\left(s_{i} f\right)\left(\gamma_{\lambda}\right)\right) T_{i} p_{\lambda}=\left(f\left(\gamma_{\lambda}\right)-\left(s_{i} f\right)\left(\gamma_{\lambda}\right)\right) d_{i}^{\sigma}\left(\gamma_{\lambda}\right) p_{\lambda} .
$$

Suppose that $a_{i}(\lambda) \neq 0$, then $s_{i} \cdot \lambda \neq \lambda$. In this case we find from Theorem 4.11 and Lemma 4.10 that $T_{i} p_{\lambda}$ is indeed of the form $A_{i, \lambda} p_{\lambda}+B_{i, \lambda} p_{s_{i} \cdot \lambda}$ with $A_{i, \lambda}=d_{i}^{\sigma}\left(\gamma_{\lambda}\right)$. In order to find $B_{i, \lambda}$ we use the intertwiner $S_{i}$. By Lemma 4.14 we have $S_{i} p_{\lambda}=g_{i, \lambda} p_{s_{i} \cdot \lambda}$ where

$$
g_{i, \lambda}= \begin{cases}1, & a_{i}(\lambda)>0 \\ q_{i}\left(\gamma_{s_{i} \cdot \lambda}\right), & a_{i}(\lambda)<0\end{cases}
$$


On the other hand, using $S_{i}=\left[T_{i}, a_{i}(Y)\right]$ we have

$$
S_{i} p_{\lambda}=\left(a_{i}\left(\gamma_{\lambda}\right)-a_{i}\left(\gamma_{s_{i} \cdot \lambda}\right)\right) B_{i, \lambda} p_{s_{i} \cdot \lambda}
$$

hence $B_{i, \lambda}=g_{i, \lambda} / 2 a_{i}\left(\gamma_{\lambda}\right)$.

Now suppose that $a_{i}(\lambda)=0$, then Theorem 4.11 and (4.4) imply that $T_{i} p_{\lambda}=d_{i}^{\sigma}\left(\gamma_{\lambda}\right) p_{\lambda}$. Since $s_{i} \cdot \lambda=\lambda$ it follows from the definition of $\gamma_{\lambda}$ that $a_{i}\left(\gamma_{\lambda}\right)=t$ for $i \in[1, n-1]$ and $a_{n}\left(\gamma_{\lambda}\right)=2 t_{0}+2 t_{n}$. From the explicit expression for $d_{i}^{\sigma}(x)$ we then find $d_{i}^{\sigma}\left(\gamma_{\lambda}\right)=\chi_{i}$.

From Lemma 4.15 and Theorem 4.11 we obtain the following result.

Proposition 4.16. The center of $H$ is equal to $\mathcal{P}_{Y}^{W_{0}}$.

Proof. From Proposition 3.14 it follows directly that the subalgebra $\mathcal{P}_{Y}^{W_{0}}$ commutes with generators $T_{i}$ of $H$, hence $\mathcal{P}_{Y}^{W_{0}}$ is contained in the center of the algebra $H$.

Let $Z$ be an element in the center of $H$. By Corollary 3.15 we may write $Z=\sum_{w \in W_{0}} T_{\bar{w}} f_{w}(Y)$ with $f_{w}(Y) \in \mathcal{P}_{Y}$. Since $Z$ commutes with $\mathcal{P}_{Y}, Z$ acts as a constant on $p_{\lambda}$, hence

$$
\sum_{w \in W_{0}} f_{w}\left(\gamma_{\lambda}\right) T_{\bar{w}} p_{\lambda}=c p_{\lambda}
$$

for some nonzero constant $c$. By Lemma 4.15 we have $T_{\bar{w}} p_{\lambda}=\sum_{v \leq w} c_{w v}^{\lambda} p_{v \cdot \lambda}$ for certain coefficients $c_{w v}^{\lambda} \in \mathbb{C}$, and $c_{w w}^{\lambda} \neq 0$ for infinitely many $\lambda \in \Lambda$. This gives

$$
\sum_{\substack{v, w \in W_{0} \\ v \leq w}} f_{w}\left(\gamma_{\lambda}\right) c_{w v}^{\lambda} p_{v \cdot \lambda}=c p_{\lambda}
$$

which implies that for $v \neq 1$

$$
\sum_{\substack{w \in W_{0} \\ w \geq v}} f_{w}\left(\gamma_{\lambda}\right) c_{w v}^{\lambda}=0 .
$$

Let $u \neq 1$ be a maximal element such that $f_{u} \neq 0$, then $c_{u u}^{\lambda}=0$ for infinitely many $\lambda \in \Lambda$. We conclude that only $f_{1}$ is nonzero. Being a central element, $Z=f_{1}(Y)$ commutes with every intertwiner $S_{i}, i \in[1, n]$. Lemma 4.5 then implies that $s_{i} f_{1}=f_{1}$, hence $f_{1}(Y) \in \mathcal{P}_{Y}^{W_{0}}$.

Theorem 4.17. The decomposition

$$
\mathcal{P}=\bigoplus_{\lambda \in \Lambda^{+}} \mathcal{P}(\lambda)
$$

is the multiplicity-free, irreducible decomposition of $\mathcal{P}$ as a $\pi(H)$-module. Moreover, (4.5) gives the decomposition of $\mathcal{P}$ into isotypical components under the action of the center $\mathcal{P}_{Y}^{W_{0}}$ of $H$, and the central character is given by $\chi_{\lambda}(p(Y))=p\left(\gamma_{\lambda}\right)$ for $p(Y) \in \mathcal{P}_{Y}^{W_{0}}$. 


\section{Nonsymmetric multivariable Wilson polynomials}

In this section we derive a few important properties for the nonsymmetric multivariable Wilson polynomials, such as orthogonality relations, evaluation formulas and the duality property. These properties are obtained in the same way as in the case of the Koornwinder polynomials, see [14,16-18].

\subsection{Duality}

We write $x_{\lambda}=\gamma_{\lambda}\left(\mathbf{t}^{\sigma}\right), \lambda \in \Lambda$, for the spectrum of the operators $Y^{\sigma} \in \mathcal{H}_{\sigma}$. Here the dual multiplicity function $\mathbf{t}^{\sigma}$ is defined by (3.8). We define evaluation mappings Ev $: \mathcal{H} \rightarrow \mathbb{C}$ and $\widetilde{\mathrm{Ev}}: \mathcal{H}_{\sigma} \rightarrow \mathbb{C}$ by

$$
\begin{array}{ll}
\operatorname{Ev}(Z)=(Z(1))\left(-x_{0}\right), & Z \in \mathcal{H}, \\
\widetilde{\operatorname{Ev}}(\widetilde{Z})=(\widetilde{Z}(1))\left(-\gamma_{0}\right), \quad \widetilde{Z} \in \mathcal{H}_{\sigma} .
\end{array}
$$

The two evaluation mappings are related via the duality anti-isomorphism $\psi: \mathcal{H} \rightarrow \mathcal{H}_{\sigma}$ from Proposition 3.11 by

$$
\operatorname{Ev}(Z)=\widetilde{\operatorname{Ev}}(\psi(Z)), \quad Z \in \mathcal{H} .
$$

Indeed, for $Z=f(X) T_{w} g(Y)$ with $f, g \in \mathcal{P}$ and $w=s_{i_{1}} \cdots s_{i_{r}} \in W_{0}$ a reduced expression, we have, using $\psi\left(X_{i}\right)=-Y_{i}^{\sigma}$ and $\psi\left(T_{i}\right)=T_{i}^{\sigma}$ for $i \in[1, n]$,

$$
\begin{aligned}
\widetilde{\operatorname{Ev}}(\psi(Z)) & =\left(g\left(-X^{\sigma}\right) T_{w^{-1}}^{\sigma} f\left(-Y^{\sigma}\right)(1)\right)\left(-\gamma_{0}\right) \\
& =\chi_{\sigma}\left(T_{w^{-1}}^{\sigma}\right) f\left(-x_{0}\right) g\left(\gamma_{0}\right) \\
& =\left(f(X) T_{w} g(Y)(1)\right)\left(-x_{0}\right) \\
& =\operatorname{Ev}(Z) .
\end{aligned}
$$

Here we use the reduced expression $w^{-1}=s_{i_{r}} \cdots s_{i_{1}}$, and $\chi$ is the trivial representation of $H_{0}$. Note that $\chi\left(T_{w}\right)=\chi_{\sigma}\left(T_{w^{-1}}\right)$ for any word $w \in W_{0}$ (and $\chi_{\sigma}: H_{0}^{\sigma} \rightarrow \mathbb{C}$ is defined in the same way as $\left.\chi: H_{0} \rightarrow \mathbb{C}\right)$. By the PBW-property for $\mathcal{H}$ from Corollary 3.15 we then see that (5.1) holds for all $Z \in \mathcal{H}$.

With the evaluation mappings and the duality anti-isomorphism $\psi: \mathcal{H} \rightarrow$ $\mathcal{H}_{\sigma}$ we construct two pairings $B: \mathcal{H} \times \mathcal{H}_{\sigma} \rightarrow \mathbb{C}$ and $\widetilde{B}: \mathcal{H}_{\sigma} \times \mathcal{H} \rightarrow \mathbb{C}$ by

$$
B(Z, \widetilde{Z})=\operatorname{Ev}\left(\psi_{\sigma}(\widetilde{Z}) Z\right), \quad \widetilde{B}(\widetilde{Z}, Z)=\widetilde{\operatorname{Ev}}(\psi(Z) \widetilde{Z})
$$

where $Z \in \mathcal{H}$ and $\widetilde{Z} \in \mathcal{H}_{\sigma}$. We have the following properties for these pairings.

Proposition 5.1. Let $Z, Z_{1}, Z_{2} \in \mathcal{H}, \widetilde{Z}, \widetilde{Z}_{1}, \widetilde{Z}_{2} \in \mathcal{H}_{\sigma}$ and $p \in \mathcal{P}$. Then

(i) $B(Z, \widetilde{Z})=\widetilde{B}(\widetilde{Z}, Z)$;

(ii) $B\left(Z_{1} Z_{2}, \widetilde{Z}\right)=B\left(Z_{2}, \psi\left(Z_{1}\right) \widetilde{Z}\right)$ and $B\left(Z, \widetilde{Z}_{1} \widetilde{Z}_{2}\right)=B\left(\psi_{\sigma}\left(\widetilde{Z}_{1}\right) Z, \widetilde{Z}_{2}\right)$;

(iii) $B((Z p)(X), \widetilde{Z})=B(Z p(X), \widetilde{Z})$ and $B\left(Z,(\widetilde{Z} p)\left(X^{\sigma}\right)\right)=B\left(Z, \widetilde{Z} p\left(X^{\sigma}\right)\right)$.

Here $p(X)$ is the multiplication operator $(p(X) f)(x)=p(x) f(x)$ for $f \in \mathcal{P}$. 
Proof. Properties (i) and (ii) follow from (5.1). Property (iii) is an immediate consequence of the identity $((Z p)(X))(1)=Z p=Z(p(X)(1))$ in $\mathcal{P}$.

The evaluation $\operatorname{Ev}\left(p_{\lambda}(X)\right)=p_{\lambda}\left(-x_{0}\right)$ of the nonsymmetric Wilson polynomial is nonzero for generic parameters $\mathbf{t}=\left(t_{0}, u_{0}, t, t_{n}, u_{n}\right)$. Indeed, for $t=0$, the polynomial $p_{\lambda}$ becomes a product of one-variable nonsymmetric Wilson polynomials $p_{\phi\left(\lambda_{i}\right)}$ which are all nonzero at $-x_{0, i}=-\left(t_{n}+u_{n}\right)$, see $[8$, Proposition 3.14]. In the next subsection we determine an explicit expression for $p_{\lambda}\left(-x_{0}\right)$.

Definition 5.2. For $\lambda \in \Lambda$ we write $E\left(x, \gamma_{\lambda} ; \mathbf{t}\right)=E\left(x, \gamma_{\lambda}\right)$ for the constant multiple of the nonsymmetric Wilson polynomial $p_{\lambda}(x ; \mathbf{t})$ that takes the value 1 at $x=-x_{0}$.

As a consequence of Proposition 5.1 we obtain

$$
B\left(p\left(X^{\sigma}\right), E\left(X, \gamma_{\lambda}\right)\right)=B\left(1,\left(p(-Y) E\left(\cdot, \gamma_{\lambda}\right)\right)(X)\right)=p\left(-\gamma_{\lambda}\right) B\left(1, E\left(X, \gamma_{\lambda}\right)\right)
$$

for any $p \in \mathcal{P}$ and $\lambda \in \Lambda$. Note that $B\left(1, E\left(X, \gamma_{\lambda}\right)=\operatorname{Ev}\left(E\left(X, \gamma_{\lambda}\right)\right)=1\right.$, so we have

$$
f\left(-\gamma_{\lambda}\right)=B\left(f\left(X^{\sigma}\right), E\left(X, \gamma_{\lambda}\right)\right), \quad g\left(-x_{\mu}\right)=\widetilde{B}\left(g(X), E_{\sigma}\left(X^{\sigma}, x_{\mu}\right)\right),
$$

for $f, g \in \mathcal{P}$ and $\lambda, \mu \in \Lambda$. The second identity is derived in the same way as the first. This immediately leads to the duality property for the renormalized nonsymmetric Wilson polynomials.

Theorem 5.3. For $\lambda, \mu \in \Lambda$ the renormalized nonsymmetric Wilson polynomials satisfy the duality property

$$
E\left(-x_{\mu}, \gamma_{\lambda} ; \mathbf{t}\right)=E\left(-\gamma_{\lambda}, x_{\mu} ; \mathbf{t}^{\sigma}\right)
$$

Proof. This follows from Proposition 5.1(i) if we set $f=E_{\sigma}\left(\cdot, x_{\mu}\right)$ and $g=E\left(\cdot, \gamma_{\lambda}\right)$ in $(5.2)$.

As a consequence of the duality property we obtain that the actions of $T_{i}$, $i \in[1, n]$, and $U_{n}$ on the renormalized nonsymmetric Wilson polynomials can be written as difference-reflection operators acting on the spectral parameters.

Proposition 5.4. Let $\lambda \in \Lambda$, then

$$
\begin{aligned}
& \left(U_{n} E\left(\cdot, \gamma_{\lambda}\right)\right)(x)=c_{0}^{\sigma}\left(-\gamma_{\lambda}\right) E\left(x, \gamma_{s_{0} \cdot \lambda}\right)-d_{0}^{\sigma}\left(-\gamma_{\lambda}\right) E\left(x, \gamma_{\lambda}\right), \\
& \left(T_{i} E\left(\cdot, \gamma_{\lambda}\right)\right)(x)=c_{i}^{\sigma}\left(-\gamma_{\lambda}\right) E\left(x, \gamma_{s_{i} \cdot \lambda}\right)-d_{i}^{\sigma}\left(-\gamma_{\lambda}\right) E\left(x, \gamma_{\lambda}\right), \quad i \in[1, n] .
\end{aligned}
$$

Proof. It is enough to prove the result for $x=-x_{\mu}$ for all $\mu \in \Lambda$. By (5.2) and Proposition 5.1 we have for $\lambda, \mu \in \Lambda$

$$
\widetilde{B}\left(E\left(X, \gamma_{\lambda}\right),\left(T_{i}^{\sigma} E_{\sigma}\left(\cdot, x_{\mu}\right)\right)\left(X^{\sigma}\right)\right)= \begin{cases}\left(U_{n} E\left(\cdot, \gamma_{\lambda}\right)\right)\left(-x_{\mu}\right), & i=0, \\ \left(T_{i} E\left(\cdot, \gamma_{\lambda}\right)\right)\left(-x_{\mu}\right), & i \in[1, n] .\end{cases}
$$


Writing out $T_{i}^{\sigma} E_{\sigma}\left(\cdot, x_{\mu}\right)$ we find using Proposition 5.1

$$
\begin{aligned}
\widetilde{B} & \left(E\left(X, \gamma_{\lambda}\right),\left(T_{i}^{\sigma} E_{\sigma}\left(\cdot, x_{\mu}\right)\right)\left(X^{\sigma}\right)\right) \\
& =\widetilde{B}\left(E\left(X, \gamma_{\lambda}\right), c_{i}^{\sigma}\left(X^{\sigma}\right)\left(s_{i} E_{\sigma}\left(\cdot, x_{\mu}\right)\right)\left(X^{\sigma}\right)-d_{i}^{\sigma}\left(X^{\sigma}\right) E_{\sigma}\left(X^{\sigma}, x_{\mu}\right)\right) \\
& =B\left(c_{i}^{\sigma}\left(X^{\sigma}\right)\left(s_{i} E_{\sigma}\left(\cdot, x_{\mu}\right)\right)(X)-d_{i}^{\sigma}\left(X^{\sigma}\right) E_{\sigma}\left(X^{\sigma}, x_{\mu}\right), E\left(X, \gamma_{\lambda}\right)\right) \\
& =c_{i}^{\sigma}\left(-\gamma_{\lambda}\right)\left(s_{i} E_{\sigma}\left(\cdot, x_{\mu}\right)\right)\left(-\gamma_{\lambda}\right)-d_{i}^{\sigma}\left(-\gamma_{\lambda}\right) E_{\sigma}\left(-\gamma_{\lambda}, x_{\mu}\right) \\
& =c_{i}^{\sigma}\left(-\gamma_{\lambda}\right) E\left(-x_{\mu}, \gamma_{s_{i} \cdot \lambda}\right)-d_{i}^{\sigma}\left(-\gamma_{\lambda}\right) E\left(-x_{\mu}, \gamma_{\lambda}\right) .
\end{aligned}
$$

The last two lines follow from (5.2), Lemma 4.10 and the duality property for the nonsymmetric Wilson polynomials, Theorem 5.3.

If $a_{i}(\lambda) \neq 0$ we already know from Lemma 4.14 that $S_{i} E\left(\cdot, \gamma_{\lambda}\right)=c E\left(\cdot, \gamma_{s_{i} \cdot \lambda}\right)$ for some nonzero constant $c$. Using Proposition 5.4 the constant $c$ can be calculated.

Corollary 5.5. Let $i \in[0, n]$, and let $\lambda \in \Lambda$ such that $s_{i} \cdot \lambda \neq \lambda$, then

$$
S_{i} E\left(\cdot, \gamma_{\lambda}\right)=2 a_{i}\left(\gamma_{\lambda}\right) c_{i}^{\sigma}\left(-\gamma_{\lambda}\right) E\left(\cdot, \gamma_{s_{i} \cdot \lambda}\right)
$$

\subsection{The evaluation formula}

Using Corollary 5.5 we can determine the value of $p_{\lambda}\left(-x_{0}\right)$.

Theorem 5.6. For $\lambda \in \Lambda$,

$$
p_{\lambda}\left(-x_{0}\right)=\prod_{\alpha \in \mathcal{R}_{r}^{+} \cap u_{\lambda}^{-1} \mathcal{R}_{r}^{-}} K_{\alpha}\left(-\gamma_{0}\right)
$$

where

$$
K_{\alpha}(x)=2 \alpha(-x) c_{\alpha}^{\sigma}(x) .
$$

Proof. By the definition of $E\left(x, \gamma_{\lambda}\right)$ we have $p_{\lambda}=p_{\lambda}\left(-x_{0}\right) E\left(\cdot, \gamma_{\lambda}\right)$. Recall that $p_{\lambda}=S_{u_{\lambda}} 1$, and let $u_{\lambda}=s_{i_{1}} \cdots s_{i_{r}}$ be a reduced expression. Using Corollary 5.5 we now find

$$
p_{\lambda}\left(-x_{0}\right)=\prod_{k=1}^{r} K_{a_{i_{k}}}\left(-\gamma_{\left(s_{i_{k+1}} \cdots s_{i_{r}}\right) \cdot 0}\right) .
$$

Recall from Lemma 4.7 that $u_{\lambda}$ is the unique shortest element in $W$ such that $u_{\lambda} \cdot 0=\lambda$, so $\left(s_{i_{k}} s_{i_{k+1}} \cdots s_{i_{r}}\right) \cdot 0 \neq\left(s_{i_{k+1}} \cdots s_{i_{r}}\right) \cdot 0$. By Lemma 4.10 we have $K_{\alpha}\left(-\gamma_{w \cdot 0}\right)=K_{w^{-1} \alpha}\left(-\gamma_{0}\right)$ for $w \in W$, so we can write $p_{\lambda}\left(-x_{0}\right)$ as a product over the set $S=\left\{\beta_{1}, \ldots, \beta_{r}\right\}$ with $\beta_{k}=s_{i_{r}} \cdots s_{i_{k+1}} a_{i_{k}}$ for $k \in[1, r]$. It is well known that the set $S$ is equal to $\mathcal{R}_{r}^{+} \cap u_{\lambda}^{-1} \mathcal{R}_{r}^{-}$, which proves the result.

\subsection{Orthogonality relations}

With the multiplicity function $\mathbf{t}$ given by $(2.2)$ we associate the Wilson parameters $a, b, c, d$ given by

$$
(a, b, c, d)=\left(t_{n}+u_{n}, t_{n}-u_{n}, t_{0}+u_{0}+\frac{1}{2}, t_{0}-u_{0}+\frac{1}{2}\right) .
$$


We assume from here on that $a, b, c, d, t>0$. We define $\Delta(\cdot)=\Delta(\cdot ; \mathbf{t})$ and $\Delta_{+}(\cdot)=\Delta_{+}(\cdot ; \mathbf{t})$ by

$$
\Delta_{+}(x ; \mathbf{t})=\prod_{1 \leq j<k \leq n} \frac{\Gamma\left(t \pm x_{j} \pm x_{k}\right)}{\Gamma\left( \pm x_{j} \pm x_{k}\right)} \prod_{j=1}^{n} \frac{\Gamma\left(a \pm x_{j}\right) \Gamma\left(b \pm x_{j}\right) \Gamma\left(c \pm x_{j}\right) \Gamma\left(d \pm x_{j}\right)}{\Gamma\left( \pm 2 x_{j}\right)}
$$

and

$$
\Delta(x ; \mathbf{t})=c_{+}(x ; \mathbf{t}) \Delta_{+}(x ; \mathbf{t})
$$

where

$$
\begin{aligned}
c_{+}(x ; \mathbf{t}) & =\prod_{\alpha \in \Sigma^{-}} c_{\alpha}(x ; \mathbf{t}) \\
& =\prod_{1 \leq j<k \leq n} \frac{\left(t-x_{j}+x_{k}\right)\left(t-x_{j}-x_{k}\right)}{\left(x_{k}+x_{j}\right)\left(x_{k}-x_{j}\right)} \prod_{j=1}^{n} \frac{\left(a-x_{j}\right)\left(b-x_{j}\right)}{-2 x_{j}}
\end{aligned}
$$

To the weight functions $\Delta$ and $\Delta_{+}$we associate two nondegenerate bilinear forms on $\mathcal{P}$ by

$$
\begin{aligned}
\langle f, g\rangle_{\mathbf{t}} & =\int_{(\mathrm{i} \mathbb{R})^{n}} f(x) g(x) \Delta(x ; \mathbf{t}) \mathrm{d} x, \\
\langle f, g\rangle_{\mathbf{t}}^{+} & =\int_{(\mathrm{i} \mathbb{R})^{n}} f(x) g(x) \Delta_{+}(x ; \mathbf{t}) \mathrm{d} x,
\end{aligned}
$$

where $d x=(2 \pi \mathrm{i})^{-n} d x_{1} d x_{2} \cdots d x_{n}$, and $\mathrm{i} \mathbb{R}$ has the standard orientation. The constant terms can be given explicitly;

$$
\langle 1,1\rangle_{\mathbf{t}}^{+}=2^{n} n ! \prod_{j=1}^{n} \frac{\Gamma(t j) \prod_{1 \leq k<l \leq 4} \Gamma\left(v_{k}+v_{l}+(j-1) t\right)}{\Gamma(t) \Gamma\left(v_{1}+v_{2}+v_{3}+v_{4}+(n+j-2) t\right)},
$$

where $\left(v_{1}, v_{2}, v_{3}, v_{4}\right)=(a, b, c, d)$, see [9]. From Stirling's formula it follows that the polynomials are integrable on $(i \mathbb{R})^{n}$ with respect to both $\Delta$ and $\Delta_{+}$. Let us remark that the weight $\Delta_{+}$is positive on $(i \mathbb{R})^{n}$ under the current conditions on $\mathbf{t}$.

Let $\iota: \mathcal{H} \rightarrow \mathcal{H}$ be the anti-isomorphism defined by $\iota\left(T_{i}\right)=T_{i}, i \in[0, n]$, and $\iota\left(X_{j}\right)=X_{j}, j \in[1, n]$.

Lemma 5.7. Let $f, g \in \mathcal{P}$ and $Z \in \mathcal{H}$, then $\langle Z f, g\rangle_{\mathbf{t}}=\langle f, \iota(Z) g\rangle_{\mathbf{t}}$.

Proof. It suffices to show that the generators $T_{i}$ and $X_{j}$ are symmetric with respect to $\langle\cdot, \cdot\rangle_{\mathrm{t}}$. For the $X_{j}$ 's this is obvious, so we only need to verify it for the $T_{i}$ 's. Writing out $\left\langle T_{i} f, g\right\rangle_{\mathbf{t}}$ we see that the proof of the lemma boils down to proving the following identity:

$$
\int_{(\mathbb{R} \mathbb{R})^{n}}\left(s_{i} f\right)(x) g(x) c_{i}(x) \Delta(x) \mathrm{d} x=\int_{(i \mathbb{R})^{n}} f(x)\left(s_{i} g\right)(x) c_{i}(x) \Delta(x) \mathrm{d} x .
$$


Using the definitions of $c_{i}, 0=1, \ldots, n$, and $\Delta$ one checks that the function $c_{i}(x) \Delta(x)$ is invariant under the action of $s_{i}$. Now for $i=1, \ldots, n$ the required identity (5.4) follows from substituting $x \mapsto s_{i} x$ on the left hand side. For $i=0$ we denote $y=\left(x_{2}, \ldots, x_{n}\right)$ and $\alpha(x)=\alpha\left(x_{1}, y\right)$ for any function $\alpha$ depending on $x_{1}, \ldots, x_{n}$. We substitute $x_{1} \mapsto 1-y_{1}$ in the left hand side of (5.4), then this becomes

$$
\int_{y_{1} \in 1+\mathrm{i} \mathbb{R}} \int_{y \in(\mathrm{i} \mathbb{R})^{n-1}} f\left(y_{1}, y\right) g\left(1-y_{1}, y\right) c_{0}\left(y_{1}, y\right) \Delta\left(y_{1}, y\right) \mathrm{d} y \mathrm{~d} y_{1} .
$$

The function $y_{1} \mapsto c_{0}\left(y_{1}, y\right) \Delta\left(y_{1}, y\right)$ has no poles inside the strip $\{0 \leq$ $\left.\Re\left(y_{1}\right) \leq 1\right\}$ due to the conditions on the parameters. Furthermore, using Stirling's formula it follows that the integral over the line segment $\left\{y_{1}=\right.$ $x+i B \mid 0 \leq x \leq 1\}$ vanishes for $B \rightarrow \pm \infty$. By Cauchy's theorem we may then shift the contour $1+i \mathbb{R}$ to $i \mathbb{R}$ without changing the outcome of the integral. This proves identity (5.4) for $i=0$.

The $Y$-operators satisfy $\iota\left(Y_{i}\right)=Y_{i}, i \in[1, n]$. From the previous lemma we then immediately obtain orthogonality relations for the Wilson polynomials. Combining this with Theorem 4.12 we have the following result.

Theorem 5.8. The nonsymmetric Wilson polynomials form an orthogonal basis for $\mathcal{P}$ with respect to $\langle\cdot, \cdot\rangle_{\mathbf{t}}$, i.e.,

$$
\left\langle p_{\lambda}, p_{\mu}\right\rangle_{\mathbf{t}}=0, \quad \lambda \neq \mu .
$$

Remark 5.9. There is a fundamental difference with the DAHA of type $\left(C^{\vee}, C\right)$. In that algebra the identity $\iota\left(Y_{i}\right)=Y_{i}$ is not valid for the corresponding $Y$-operators. Therefore, the polynomial eigenfunctions of the $Y$-operators, the nonsymmetric Koornwinder polynomials, satisfy biorthogonality relations, see $[16]$.

Next we compute the diagonal terms $\left\langle E\left(\cdot, \gamma_{\lambda}\right), E\left(\cdot, \gamma_{\lambda}\right)\right\rangle_{\mathbf{t}}$.

Lemma 5.10. For $\lambda \in \Lambda$ and $i \in[0, n]$,

$$
\left\langle E\left(\cdot, \gamma_{\lambda}\right), E\left(\cdot, \gamma_{\lambda}\right)\right\rangle_{\mathbf{t}}=\frac{c_{-a_{i}}^{\sigma}\left(\gamma_{\lambda}\right)}{c_{a_{i}}^{\sigma}\left(\gamma_{\lambda}\right)}\left\langle E\left(\cdot, \gamma_{s_{i} \cdot \lambda}\right), E\left(\cdot, \gamma_{s_{i} \cdot \lambda}\right)\right\rangle_{\mathbf{t}} .
$$

Proof. By Lemma 5.7 and the definition (4.3) of the intertwiners $S_{i}$ we have

$$
\left\langle S_{i} f, g\right\rangle_{\mathbf{t}}=-\left\langle f, S_{i} g\right\rangle_{\mathbf{t}}, \quad f, g \in \mathcal{P} .
$$

Since $S_{i} E\left(\cdot, \gamma_{\lambda}\right)=b_{\lambda, i} E\left(\cdot, \gamma_{s_{i} \cdot \lambda}\right)$, with $b_{\lambda, i}$ given in Corollary 5.5 , we have

$$
\left\langle S_{i} E\left(\cdot, \gamma_{\lambda}\right), S_{i} E\left(\cdot, \gamma_{\lambda}\right)\right\rangle_{\mathbf{t}}=b_{\lambda, i}^{2}\left\langle E\left(\cdot, \gamma_{s_{i} \cdot \lambda}\right), E\left(\cdot, \gamma_{s_{i} \cdot \lambda}\right)\right\rangle_{\mathbf{t}} .
$$

On the other hand, by Lemma 4.5 we have

$$
\begin{aligned}
\left\langle S_{i} E\left(\cdot, \gamma_{\lambda}\right), S_{i} E\left(\cdot, \gamma_{\lambda}\right)\right\rangle_{\mathbf{t}} & =-\left\langle E\left(\cdot, \gamma_{\lambda}\right), S_{i}^{2} E\left(\cdot, \gamma_{\lambda}\right)\right\rangle_{\mathbf{t}} \\
& =-q_{i}\left(\gamma_{\lambda}\right)\left\langle E\left(\cdot, \gamma_{\lambda}\right), E\left(\cdot, \gamma_{\lambda}\right)\right\rangle_{\mathbf{t}} .
\end{aligned}
$$

Now the result follows from $-b_{\lambda, i}^{2} / q_{i}\left(\gamma_{\lambda}\right)=c_{-a_{i}}^{\sigma}\left(\gamma_{\lambda}\right) / c_{a_{i}}^{\sigma}\left(\gamma_{\lambda}\right)$. 
For $\lambda \in \Lambda$ we define

$$
N\left(\gamma_{\lambda} ; \mathbf{t}\right)=\frac{1}{\langle 1,1\rangle_{\mathbf{t}}} \prod_{\alpha \in \mathcal{R}_{r}^{+} \cap u_{\lambda} \mathcal{R}_{r}^{-}} \frac{c_{-\alpha}^{\sigma}\left(\gamma_{\lambda}\right)}{c_{\alpha}^{\sigma}\left(\gamma_{\lambda}\right)}
$$

From $E\left(\cdot, \gamma_{0}\right)=1$, Lemma 5.10 and arguments similar as in the proof of Theorem 5.6 we see that $N\left(\gamma_{\lambda}\right)^{-1}$ is the 'quadratic norm' for $E\left(\cdot, \gamma_{\lambda}\right)$.

Theorem 5.11. For $\lambda \in \Lambda$,

$$
\left\langle E\left(\cdot, \gamma_{\lambda}\right), E\left(\cdot, \gamma_{\lambda}\right)\right\rangle_{\mathbf{t}}=\frac{1}{N\left(\gamma_{\lambda}\right)}
$$

\subsection{The Fourier transform}

We define

$$
\operatorname{Spec}(-Y)=\left\{-\gamma_{\lambda} \mid \lambda \in \Lambda\right\}
$$

Let $F$ be the vector space of complex-valued functions on $\operatorname{Spec}(-Y)$ with finite support. A linear basis for $F$ is formed by the functions $\delta_{\lambda}, \lambda \in \Lambda$, given by

$$
\delta_{\lambda}\left(-\gamma_{\mu}\right)= \begin{cases}0, & \mu \neq \lambda \\ 1, & \mu=\lambda\end{cases}
$$

We define a bilinear form $[\cdot, \cdot]: F \times F \rightarrow \mathbb{C}$ by

$$
[f, g]_{\mathbf{t}}=\sum_{\gamma \in \operatorname{Spec}(-Y)} f(\gamma) g(\gamma) N(-\gamma ; \mathbf{t})
$$

The functions $\delta_{\lambda}, \lambda \in \Lambda$, are orthogonal with respect to this bilinear form.

We define the polynomial Fourier transform $\mathcal{F}: \mathcal{P} \rightarrow F$ by

$$
(\mathcal{F} p)(\gamma)=\langle p, E(\cdot,-\gamma)\rangle_{\mathbf{t}}, \quad p \in \mathcal{P}, \gamma \in \operatorname{Spec}(-\mathrm{Y}) .
$$

Since the bilinear form $\langle\cdot, \cdot\rangle$ is nondegenerate, the map $\mathcal{F}$ is an injective map. Moreover, $\mathcal{F} E\left(\cdot ; \gamma_{\lambda}\right)$ is a multiple of $\delta_{\lambda}$, hence $\mathcal{F}$ maps an orthogonal basis of $\mathcal{P}$ to an orthogonal basis of $F$, which implies that $\mathcal{F}$ is surjective. We also define a linear map $\mathcal{G}: F \rightarrow \mathcal{P}$ by

$$
(\mathcal{G} g)(x)=[g, E(x,-\cdot)]_{\mathbf{t}}=\sum_{\gamma \in \operatorname{Spec}(-Y)} g(\gamma) E(x,-\gamma) N(-\gamma ; \mathbf{t}), \quad g \in F, x \in \mathbb{C}^{n} .
$$

Theorem 5.12. The map $\mathcal{G}: F \rightarrow \mathcal{P}$ is the inverse of the Fourier transform $\mathcal{F}$ : $\mathcal{P} \rightarrow F$. Moreover, we have the Plancherel-type formulas

$$
\left[\mathcal{F} f_{1}, \mathcal{F} f_{2}\right]_{\mathbf{t}}=\left\langle f_{1}, f_{2}\right\rangle_{\mathbf{t}}, \quad\left\langle\mathcal{G} g_{1}, \mathcal{G} g_{2}\right\rangle_{\mathbf{t}}=\left[g_{1}, g_{2}\right]_{\mathbf{t}},
$$

for $f_{1}, f_{2} \in \mathcal{P}$ and $g_{1}, g_{2} \in F$.

Proof. The proof is straightforward using the orthogonality relations for the nonsymmetric Wilson polynomials, see Theorems 5.8 and 5.11. 
The affine Weyl group $W$ has an action on $F$ defined by

$$
(w f)\left(-\gamma_{\lambda}\right)=f\left(-\gamma_{w^{-1} \cdot \lambda}\right), \quad w \in W, f \in F .
$$

We now define an action of $\mathcal{H}_{\sigma}$ on $F$. For $f \in F$ and $Z \in \mathcal{H}_{\sigma}$ we set

$$
(Z f)(\gamma)=(Z \bar{f})(\gamma)
$$

Here $\bar{f}$ denotes an arbitrary function $\bar{f}: \mathbb{C}^{n} \rightarrow \mathbb{C}$ such that $\bar{f}(\gamma)=f(\gamma)$ for any $\gamma \in \operatorname{Spec}(-Y)$, and the action of $\mathcal{H}_{\sigma}$ on the right hand side is given by the usual difference-reflection operators. We need to verify that (5.5) is welldefined.

Lemma 5.13. The action of $\mathcal{H}_{\sigma}$ on $F$ defined by (5.5) is independent of the choice of extension $\bar{f}$ of $f$.

Proof. It is enough to verify that the lemma is true for generators $T_{i}^{\sigma}, i \in[0, n]$, and $X_{i}, i \in[1, n]$, of the algebra $\mathcal{H}_{\sigma}$. For the $X_{i}$ 's this is obvious. Let $f \in F$ and let $\bar{f}$ be an extensions of $f$. By Lemma 4.10 we have $\left(s_{i} \bar{f}\right)(\gamma)=\left(s_{i} f\right)(\gamma)$ for $\gamma \in \operatorname{Spec}(-Y)$ and $i \in[0, n]$. For the $T_{i}^{\sigma}$ 's this gives us

$$
\left(T_{i}^{\sigma} \bar{f}\right)(\gamma)=\chi_{i} f(\gamma)+c_{i}^{\sigma}(\gamma)\left(\left(s_{i} f\right)(\gamma)-f(\gamma)\right),
$$

for any extension $\bar{f}$ of $f$. This is clearly independent of the choice of extension.

We have the following intertwining properties for the Fourier transforms $\mathcal{F}$ and $\mathcal{G}$.

Proposition 5.14. The Fourier transforms $\mathcal{F}: \mathcal{P} \rightarrow F$ and $\mathcal{G}: F \rightarrow \mathcal{P}$ have the following intertwining properties:

$$
\begin{aligned}
& \mathcal{F} \circ Z=\sigma(Z) \circ \mathcal{F}, \quad Z \in \mathcal{H}, \\
& \mathcal{G} \circ Z=\sigma_{\sigma}(Z) \circ \mathcal{G}, \quad Z \in \mathcal{H}_{\sigma} .
\end{aligned}
$$

Proof. It is enough to check the intertwining property of $\mathcal{F}$ for the generators $Y_{i}, T_{i}, U_{n}, i \in[1, n]$, of the algebra $\mathcal{H}$. This can be done using Lemma 5.7, Propositions 5.4 and 3.11 .

The intertwining property of $\mathcal{G}$ follows from the intertwining property of $\mathcal{F}$ and Theorem 5.12. Let $g \in F$, then $g=\mathcal{F} f$ for a unique $f \in \mathcal{P}$, or equivalently $f=\mathcal{G} g$. Let $Z \in \mathcal{H}_{\sigma}$, then $Z g=\mathcal{F}\left(\sigma_{\sigma}(Z) f\right)$, which gives us

$$
\mathcal{G}(Z g)=(\mathcal{G} \circ \mathcal{F})\left(\sigma_{\sigma}(Z) f\right)=\sigma_{\sigma}(Z) f=\sigma_{\sigma}(Z) \mathcal{G} g \text {. }
$$

\section{Symmetric multivariable Wilson polynomials}

In this section we define and study symmetric multivariable Wilson polynomials. They turn out to coincide with the multivariable Wilson polynomials as defined by van Diejen [21]. In [21] it is shown that the symmetric Wilson 
polynomials in $n$ variables diagonalize a system of $n$ commuting difference operators, the duality property is obtained, and orthogonality relations are given. We derive these properties here from the representation theory of the rational DAHA $\mathcal{H}$, and we provide the link with the nonsymmetric theory.

\subsection{Symmetric Wilson polynomials}

For $\lambda \in \Lambda^{+}$we define

$$
\mathcal{P}(\lambda)^{W_{0}}=\mathcal{P}(\lambda) \cap \mathcal{P}^{W_{0}} .
$$

Observe that it follows from $\mathcal{P}=\bigoplus_{\lambda \in \Lambda^{+}} \mathcal{P}(\lambda)$ that $\mathcal{P}^{W_{0}}=\bigoplus_{\lambda \in \Lambda^{+}} \mathcal{P}(\lambda)^{W_{0}}$.

Proposition 6.1. For $\lambda \in \Lambda^{+}$the space $\mathcal{P}(\lambda)^{W_{0}}$ is one-dimensional. Furthermore, any $f \in \mathcal{P}(\lambda)^{W_{0}}$ is given by

$$
f=\sum_{\mu \in W_{0} \lambda} d_{\lambda, \mu} E\left(\cdot, \gamma_{\mu}\right),
$$

with

$$
d_{\lambda, \mu}=d_{\lambda} c_{+}^{\sigma}\left(-\gamma_{\mu}\right),
$$

for some constant $d_{\lambda}$ independent of $\mu \in W_{0} \lambda$.

Proof. Let $i \in[1, n]$. From Proposition 5.4 we know that

$$
\left(T_{i}-\chi_{i}\right) E\left(\cdot, \gamma_{\mu}\right)=c_{a_{i}}^{\sigma}\left(-\gamma_{\mu}\right)\left(E\left(\cdot, \gamma_{s_{i} \mu}\right)-E\left(\cdot, \gamma_{\mu}\right)\right)
$$

if $s_{i} \mu \neq \mu$. Applying $\left(T_{i}-\chi_{i}\right)$ to the expansion (6.1) and using $\left(T_{i}-\chi_{i}\right) f=0$ we find a recurrence relation for the coefficients $d_{\lambda, \mu}$;

$$
d_{\lambda, s_{i} \mu}=\frac{c_{a_{i}}^{\sigma}\left(-\gamma_{\mu}\right)}{c_{-a_{i}}^{\sigma}\left(-\gamma_{\mu}\right)} d_{\lambda, \mu}
$$

Here we also used $c_{\alpha}\left(\gamma_{s_{i} \mu}\right)=\left(s_{i} c_{\alpha}\right)\left(\gamma_{\mu}\right)=c_{s_{i} \alpha}\left(\gamma_{\mu}\right)$. Let $s_{i_{1}} \cdots s_{i_{r}}$ be a reduced expression for $v_{\mu}$ (defined in the beginning of subsection 4.2), and define

$$
\beta_{1}=a_{i_{1}}, \quad \beta_{j}=s_{i_{j-1}} \cdots s_{i_{1}} a_{i_{j}}, \quad j=2, \ldots, r,
$$

then iterating (6.2) gives

$$
d_{\lambda, \mu}=d_{\lambda, \lambda} \prod_{j=1}^{r} \frac{c_{-\beta_{j}}^{\sigma}\left(\gamma_{\lambda}\right)}{c_{\beta_{j}}^{\sigma}\left(\gamma_{\lambda}\right)} .
$$

From the definition of $c_{+}(x)$ we see that $\left(s_{i} c_{+}\right)(x)=c_{+}(x) c_{-a_{i}}(-x) / c_{a_{i}}(-x)$, and then

$$
d_{\lambda, \mu}=d_{\lambda, \lambda} \frac{\left(v_{\mu} c_{+}^{\sigma}\right)\left(-\gamma_{\lambda}\right)}{c_{+}^{\sigma}\left(-\gamma_{\lambda}\right)}=d_{\lambda, \lambda} \frac{c_{+}^{\sigma}\left(-\gamma_{\mu}\right)}{c_{+}^{\sigma}\left(-\gamma_{\lambda}\right)} .
$$

If we now set $d_{\lambda, \lambda}=d_{\lambda} c_{+}^{\sigma}\left(-\gamma_{\lambda}\right)$, the lemma follows.

In Sect. 6.5 we give an operator that maps $\mathcal{P}(\lambda)$ onto $\mathcal{P}(\lambda)^{W_{0}}$. 
Definition 6.2. For $\lambda \in \Lambda^{+}$we define the symmetric multivariable Wilson polynomial $E^{+}\left(x, \gamma_{\lambda}\right)$ to be the unique polynomial in $\mathcal{P}(\lambda)^{W_{0}}$ that takes the value 1 at $x=x_{0}$.

Since $f\left(x_{0}\right)=f\left(-x_{0}\right)$ if $f \in \mathcal{P}^{W_{0}}$ and $E\left(-x_{0}, \gamma_{\lambda}\right)=1$, by Proposition 6.1 the symmetric Wilson polynomial $E^{+}\left(\cdot, \gamma_{\lambda}\right)$ is the polynomial in $\mathcal{P}(\lambda)^{W_{0}}$ with constant $d_{\lambda}$ given by

$$
d_{\lambda}=\left(\sum_{\mu \in W_{0} \lambda} c_{+}^{\sigma}\left(-\gamma_{\mu}\right)\right)^{-1}
$$

This constant can be evaluated with the following result.

Lemma 6.3. The function $K=\sum_{w \in W_{0}} w c_{+}$is a constant function, and

$$
K(x)=\sum_{\mu \in W_{0} \lambda} c_{+}\left(-x_{\mu}\right)=c_{+}\left(-x_{0}\right),
$$

for any $\lambda \in \Lambda^{+}$.

Proof. We define the polynomial $m$ by

$$
m(x)=\prod_{\alpha \in \Sigma^{-}} \alpha(x)=(-2)^{n} \prod_{1 \leq j<k \leq n}\left(x_{k}^{2}-x_{j}^{2}\right) \prod_{j=1}^{n} x_{j} .
$$

This is an anti-symmetric polynomial, i.e., $(w m)(x)=(-1)^{l(w)} m(x)$ for any $w \in W_{0}$, where $l(w)$ denotes the length of $w$. It is well known that any anti-symmetric polynomial is the product of $m$ with a symmetric polynomial. The product $m(x) K(x)$ is manifestly an anti-symmetric polynomial;

$$
\begin{aligned}
m(x) K(x)= & \sum_{w \in W_{0}}(-1)^{l(w)} w \\
& \times\left(\prod_{1 \leq j<k \leq n}\left(t+x_{k}-x_{j}\right)\left(t-x_{k}-x_{j}\right) \prod_{j=1}^{n}\left(a-x_{j}\right)\left(b-x_{j}\right)\right) .
\end{aligned}
$$

Expanding both $m(x)$ and $m(x) K(x)$ in monomials $x^{\mu}, \mu \in \mathbb{Z}_{>0}^{n}$, we see that any monomial with nonzero coefficient in the expansion of $m(x) K(x)$ also has nonzero coefficient in the expansion of $m(x)$, hence $K(x)$ is a constant. Now let $\lambda \in \Lambda^{+}$, then by Lemma 4.10

$$
K(x)=K\left(-x_{\lambda}\right)=\sum_{w \in W_{0}}\left(w c_{+}\right)\left(-x_{\lambda}\right)=\sum_{\substack{w \in W_{0} \\ w \lambda \neq \lambda}} c_{+}\left(-x_{w \lambda}\right)+\sum_{\substack{w \in W_{0} \\ w \lambda=\lambda}}\left(w c_{+}\right)\left(-x_{\lambda}\right) .
$$

Consider a term $\left(w c_{+}\right)\left(-x_{\lambda}\right)$ with $w \neq 1$ from the second sum. There exists a simple root $a_{i}, i \in[1, n]$, such that $\alpha=w^{-1} a_{i} \in \Sigma^{-}$. Then we see that the 
factor $\left(w c_{\alpha}\right)\left(-x_{\lambda}\right)=c_{a_{i}}\left(-x_{\lambda}\right)$ equals zero, since $a_{i}\left(x_{\lambda}\right)=t$ for $i \in[1, n-1]$ and $a_{n}\left(x_{\lambda}\right)=2 t_{n}+2 u_{n}$. This shows that

$$
K(x)=\sum_{\mu \in W_{0} \lambda} c_{+}\left(-x_{\mu}\right) .
$$

In particular, for $\lambda=0$ this gives $K(x)=c_{+}\left(-x_{0}\right)$.

\subsection{Orthogonality relations}

Next we show that the symmetric Wilson polynomials are orthogonal with respect to $\langle\cdot, \cdot\rangle_{\mathbf{t}}^{+}$. First a useful lemma. Let $V^{+} \in W$ be the primitive idempotent

$$
V^{+}=\frac{1}{\left|W_{0}\right|} \sum_{w \in W_{0}} w,
$$

where $\left|W_{0}\right|=2^{n} n$ ! is the cardinality of the finite Weyl group $W_{0}$. Note that $V^{+} f=f$ if $f \in \mathcal{P}^{W_{0}}$.

Lemma 6.4. For $f, g \in \mathcal{P}^{W_{0}}$ we have

$$
\langle f, g\rangle_{\mathbf{t}}=\frac{c_{+}\left(-x_{0}\right)}{\left|W_{0}\right|}\langle f, g\rangle_{\mathbf{t}}^{+} .
$$

Proof. First note that $s_{i}, i \in[1, n]$, is symmetric with respect to $\langle\cdot, \cdot\rangle_{\mathbf{t}}^{+}$, since $s_{i} \Delta^{+}=\Delta^{+}$, hence so is $V^{+}$. Let $f, g \in \mathcal{P}^{W_{0}}$, then by Lemma 6.3

$$
\begin{aligned}
\langle f, g\rangle_{\mathbf{t}} & =\left\langle V^{+} f, g\right\rangle_{\mathbf{t}}=\left\langle V^{+} f, c_{+} g\right\rangle_{\mathbf{t}}^{+} \\
& =\left\langle f, V^{+}\left(c_{+} g\right)\right\rangle_{\mathbf{t}}^{+}=\left\langle f,\left(V^{+} c_{+}\right) g\right\rangle_{\mathbf{t}}^{+}=\frac{c_{+}\left(-x_{0}\right)}{\left|W_{0}\right|}\langle f, g\rangle_{\mathbf{t}}^{+} .
\end{aligned}
$$

This proves the lemma.

Theorem 6.5. The symmetric Wilson polynomials $E^{+}\left(\cdot, \gamma_{\lambda}\right), \lambda \in \Lambda^{+}$, form an orthogonal basis for $\mathcal{P}^{W_{0}}$ with respect to $\langle\cdot, \cdot\rangle_{\mathbf{t}}^{+}$.

Proof. Since $\mathcal{P}^{W_{0}}=\bigoplus_{\lambda \in \Lambda^{+}} \mathcal{P}(\lambda)^{W_{0}}$ it is clear that the symmetric Wilson polynomials form a basis for $\mathcal{P}^{W_{0}}$. From the orthogonality relations for the nonsymmetric Wilson polynomials and the definition of the symmetric Wilson polynomials, it follows that

$$
\left\langle E^{+}\left(\cdot, \gamma_{\lambda}\right), E^{+}\left(\cdot, \gamma_{\mu}\right)\right\rangle_{\mathbf{t}}=0, \quad \lambda \neq \mu .
$$

Now the orthogonality with respect $\langle\cdot, \cdot\rangle_{\mathbf{t}}^{+}$follows from Lemma 6.4.

\subsection{Difference equations}

A difference-reflection operator $D \in \mathcal{P}_{Y}^{W_{0}}$ (considered as a subalgebra of $\operatorname{End}(\mathcal{P}))$ is of the form

$$
D=\sum_{\lambda \in \Lambda, w \in W_{0}} c_{\lambda, w}(x) \tau(\lambda) w,
$$


with $c_{\lambda, w}(x) \in \mathbb{C}(x)$. Restricted to the algebra of $W_{0}$-invariant polynomials, it becomes a difference operator which we denote by $D_{\text {sym }}$;

$$
D_{s y m}=\sum_{\lambda \in \Lambda, w \in W_{0}} c_{\lambda, w}(x) \tau(\lambda) .
$$

Moreover, $D_{\text {sym }}$ is $W_{0}$-invariant, i.e., $w \circ D_{\text {sym }} \circ w^{-1}=D_{\text {sym }}$ for any $w \in W_{0}$. Together with Theorem 4.17 this leads to the following property of the symmetric Wilson polynomials.

Theorem 6.6. The symmetric Wilson polynomials satisfy the difference equation

$$
f(Y)_{\text {sym }} E^{+}\left(\cdot, \gamma_{\lambda}\right)=f\left(\gamma_{\lambda}\right) E^{+}\left(\cdot, \gamma_{\lambda}\right), \quad \lambda \in \Lambda^{+},
$$

for any $f \in \mathcal{P}^{W_{0}}$.

In particular, the symmetric Wilson polynomials are eigenfunctions of the following explicit difference operator.

Proposition 6.7. The symmetric Wilson polynomials $E^{+}\left(\cdot, \gamma_{\lambda}\right), \lambda \in \Lambda^{+}$, are eigenfunctions of the second order difference operator $L$ defined by

$$
\begin{aligned}
L & =\sum_{i=1}^{n} A_{i}(x)\left(\tau\left(\epsilon_{i}\right)-1\right)+A_{i}(-x)\left(\tau\left(-\epsilon_{i}\right)-1\right), \\
A_{i}(x) & =\frac{\left(a+x_{i}\right)\left(b+x_{i}\right)\left(c+x_{i}\right)\left(d+x_{i}\right)}{2 x_{i}\left(2 x_{i}+1\right)} \prod_{j \neq i} \frac{\left(t+x_{i}+x_{j}\right)\left(t+x_{i}-x_{j}\right)}{\left(x_{i}+x_{j}\right)\left(x_{i}-x_{j}\right)},
\end{aligned}
$$

for eigenvalue $\sum_{i=1}^{n} \lambda_{i}\left(\lambda_{i}+a+b+c+d-1+2(n-i) t\right)$.

Proof. Let $f_{2} \in \mathcal{P}^{W_{0}}$ be defined by $f_{2}(x)=\sum_{i=1}^{n} x_{i}^{2}$. We show that $L=$ $\left.\left(f_{2}(Y)-f_{2}\left(\gamma_{0}\right)\right)\right|_{\text {sym }}$. Then by Theorem 4.17 the symmetric Wilson polynomials $E^{+}\left(\cdot, \gamma_{\lambda}\right)$ are eigenfunctions of $L$ for eigenvalue $\sum_{i}\left(\gamma_{\lambda, i}^{2}-\gamma_{0, i}^{2}\right)=\sum_{i} \lambda_{i}\left(\lambda_{i}+\right.$ $\left.2 \gamma_{\lambda, i}\right)$, which is precisely the eigenvalue in the proposition.

Recall the reduced expression (2.1) for $\tau\left(\epsilon_{i}\right)$, and recall that $w \tau\left(\epsilon_{i}\right) w^{-1}=$ $\tau\left(w \epsilon_{i}\right)$ for $w \in W_{0}$. From the explicit expression for the $Y$-operators we see that $Y_{i}^{2}$ expressed in terms of the generators $T_{j}$ contains only terms in which $T_{0}$ occurs at most once (in the terms in which $T_{0}$ occurs twice, the $T_{0}$ 's vanish using the quadratic relations in $\mathcal{H}$ ). Hence $L$ is of the form

$$
C(x)+\sum_{i=1}^{n} A_{i}(x) \tau\left(\epsilon_{i}\right)+B_{i}(x) \tau\left(-\epsilon_{i}\right)
$$

with $A_{i}(x), B_{i}(x), C(x) \in \mathbb{C}(x)$. Since $Y_{i} 1=\gamma_{0, i}$ and therefore $L 1=0$, we see immediately that $C(x)=-\sum_{i}\left(A_{i}(x)+B_{i}(x)\right)$. By the $W_{0}$-invariance of $L$ the coefficients $A_{i}$ and $B_{i}$ must satisfy

$$
s_{\epsilon_{i}-\epsilon_{j}} A_{i}(x)=A_{j}(x), \quad s_{2 \epsilon_{i}} A_{i}(x)=B_{i}(x) .
$$


Now it is enough to find the coefficient $A_{1}(x)$ of $\tau\left(\epsilon_{1}\right)$. We write

$$
\sum_{i=1}^{n} Y_{i}^{2}=\sum_{w \in W} c_{w}(x) w
$$

with $c_{w}(x) \in \mathbb{C}(x)$ and every $w \in W$ occurring in this expansion is an ordered subword of

$$
s_{i} s_{i+1} \cdots s_{n} s_{n-1} \cdots s_{0} s_{1} \cdots s_{i-1} \quad \text { or } \quad s_{i-1} s_{i-2} \cdots s_{0} s_{1} \cdots s_{n} s_{n-1} \cdots s_{i},
$$

for $i \in[1, n]$. If we write here $s_{0}=s_{1} \cdots s_{n} \cdots s_{1} \tau\left(\epsilon_{1}\right)$, and we use $u \tau\left(\epsilon_{i}\right)=$ $\tau\left(u \epsilon_{i}\right) u, u \in W_{0}$, to write every $w$ in (6.3) as $\tau\left( \pm \epsilon_{i}\right) v$ for some $v \in W_{0}$, then we see that $\tau\left(\epsilon_{1}\right)$ occurs only once. The only contribution to $\tau\left(\epsilon_{1}\right)$ comes from the expression $T_{1} \cdots T_{n} \cdots T_{0}$ in $Y_{1}^{2}$, and this gives us

$$
A_{1}(x)=c_{\epsilon_{1}-\epsilon_{2}}(x) \cdots c_{\epsilon_{1}-\epsilon_{n}}(x) c_{2 \epsilon_{1}}(x) c_{\epsilon_{1}+\epsilon_{n}} \cdots c_{\epsilon_{1}+\epsilon_{2}}(x) c_{\delta+2 \epsilon_{1}}(x) .
$$

Now the result follows from writing out this expression.

Remark 6.8. It can be shown that the difference operator $L$ acts triangularly with respect to the dominance ordering on the $W_{0}$-symmetric monomial $m_{\lambda}=$ $\sum_{\mu \in \mathcal{S}_{n} \lambda} x^{\phi(\mu)}, \lambda \in \Lambda^{+}$;

$$
L m_{\lambda}=\gamma_{\lambda}^{+} m_{\lambda}+\sum_{\mu<\lambda} c_{\lambda \mu} m_{\mu},
$$

with $\gamma_{\lambda}^{+}$the eigenvalue from Proposition 6.7. This shows that the eigenspace of $L$ for eigenvalue $\gamma_{\lambda}^{+}$is one-dimensional. The multivariable Wilson polynomials defined by van Diejen [20,21], are eigenfunctions of $L$ for eigenvalue $\gamma_{\lambda}^{+}$, hence they coincide with our symmetric Wilson polynomials.

\subsection{Duality}

Using $f(Y) E^{+}\left(\cdot, \gamma_{\lambda}\right)=f\left(\gamma_{\lambda}\right) E^{+}\left(\cdot, \gamma_{\lambda}\right)$ for $f \in \mathcal{P}^{W_{0}}$, the duality property for the symmetric Wilson polynomials can be obtained in the same way as for the nonsymmetric ones, see Theorem 5.3.

Theorem 6.9. For $\lambda, \mu \in \Lambda^{+}$, we have

$$
E^{+}\left(x_{\mu}, \gamma_{\lambda}\right)=E_{\sigma}^{+}\left(\gamma_{\lambda}, x_{\mu}\right) \text {. }
$$

Using the duality property, the difference equations from Theorem 6.6 give rise to recurrence relations for the symmetric Wilson polynomials.

\subsection{The symmetrizer}

We define an operator $C^{+}: \mathbb{C}(x) \rightarrow \mathbb{C}(x)$ by

$$
C^{+}=V^{+} c_{+}(X)
$$

where $c_{+}(X)$ is multiplication by $c_{+}(x)$, see (5.3). We call $C^{+}$the symmetrizer.

Lemma 6.10. The symmetrizer $C^{+}$has the following properties:

(i) For $f \in \mathcal{P}$ we have $C^{+} f \in \mathcal{P}^{W_{0}}$. 
(ii) For $f \in \mathcal{P}^{W_{0}}$ we have $C^{+} f=\left|W_{0}\right|^{-1} c_{+}\left(-x_{0}\right) f$.

(iii) For $i \in[1, n], C^{+} T_{i}=\chi_{i} C^{+}=T_{i} C^{+}$.

Proof. (i) Let us denote $\left|W_{0}\right|\left(C^{+} f\right)(x)=K_{f}(x)$. In the same way as in the proof of Lemma 6.3 we find that the product $m(x) K_{f}(x)$ is an anti-symmetric polynomial, from which it follows that $K_{f} \in \mathcal{P}^{W_{0}}$.

(ii) If $f \in \mathcal{P}^{W_{0}}$ we see that $K_{f}=f K_{1}$. Then the result follows from Lemma 6.3.

(iii) Let $i \in[1, n]$. Using $T_{i}=\chi_{i}+c_{i}(X)\left(s_{i}-1\right)$ and $c_{a_{i}}+c_{-a_{i}}=2 \chi_{i}$ we find $T_{i}+\chi_{i}=\left(s_{i}+1\right) c_{-a_{i}}(X)$, since $s_{i} c_{a_{i}}=c_{s_{i} a_{i}}$. Furthermore, from the definition of $c_{+}(x)$ it follows that $s_{i} c_{+}(X) c_{a_{i}}(X)=c_{+}(X) s_{i} c_{-a_{i}}(X)$. This gives

$$
\begin{aligned}
C^{+}\left(T_{i}+\chi_{i}\right) & =V^{+} c_{+}(X) s_{i} c_{-a_{i}}(X)+V^{+} c_{+}(X) c_{-a_{i}}(X) \\
& =V^{+} s_{i} c_{+}(X) c_{a_{i}}(X)+V^{+} c_{+}(X) c_{-a_{i}}(X) \\
& =V^{+} c_{+}(X) 2 \chi_{i} \\
& =2 \chi_{i} C^{+}
\end{aligned}
$$

since $V^{+} s_{i}=V^{+}$. This proves the identity $C^{+} T_{i}=\chi_{i} C^{+}$. The identity $T_{i} C^{+}=\chi_{i} C^{+}$follows from (i), since $T_{i} f=\chi_{i} f$ for $f \in \mathcal{P}^{W_{0}}$.

We show that the symmetrizer $C^{+}$maps $\mathcal{P}(\lambda)$ onto $\mathcal{P}(\lambda)^{W_{0}}$ for all $\lambda \in$ $\Lambda^{+}$.

Proposition 6.11. For $\lambda \in \Lambda^{+}$and $\mu \in W_{0} \lambda$, we have

$$
C^{+} E\left(\cdot, \gamma_{\mu}\right)=\frac{c_{+}\left(-x_{0}\right)}{\left|W_{0}\right|} E^{+}\left(\cdot, \gamma_{\lambda}\right) \text {. }
$$

Proof. Let $\lambda \in \Lambda^{+}$and $\mu \in W_{0} \lambda$. We know that $C^{+} E\left(\cdot, \gamma_{\mu}\right) \in \mathcal{P}^{W_{0}}$, so by Theorem 6.5 there exists an expansion

$$
C^{+} E\left(\cdot, \gamma_{\mu}\right)=\sum_{\nu \in \Lambda^{+}} b_{\mu, \nu} E^{+}\left(\cdot, \gamma_{\nu}\right)
$$

Using the orthogonality we find

$$
\begin{aligned}
b_{\mu, \nu}\left\langle E^{+}\left(\cdot, \gamma_{\nu}\right), E^{+}\left(\cdot, \gamma_{\nu}\right)\right\rangle_{\mathbf{t}}^{+} & =\left\langle E^{+}\left(\cdot, \gamma_{\nu}\right), C^{+} E\left(\cdot, \gamma_{\mu}\right)\right\rangle_{\mathbf{t}}^{+} \\
& =\left\langle V^{+} E^{+}\left(\cdot, \gamma_{\nu}\right), c_{+}(x) E\left(\cdot, \gamma_{\mu}\right)\right\rangle_{\mathbf{t}}^{+} \\
& =\left\langle E^{+}\left(\cdot, \gamma_{\nu}\right), E\left(\cdot, \gamma_{\mu}\right)\right\rangle_{\mathbf{t}} .
\end{aligned}
$$

Since $E^{+}\left(\cdot, \gamma_{\nu}\right) \in \mathcal{P}(\nu)^{W_{0}}$ we obtain from the orthogonality relations for the nonsymmetric Wilson polynomials that $b_{\mu, \nu}=0$ if $\mu \notin W_{0} \nu$, i.e., if $\nu \neq \lambda$. We can find $b_{\mu, \lambda}$ by evaluating at $x_{0}$. Using $\left(w c_{+}\right)\left(-x_{0}\right)=0$ if $w \neq 1$, we obtain

$$
\begin{aligned}
\left(C^{+} E\left(\cdot, \gamma_{\mu}\right)\right)\left(x_{0}\right) & =\left|W_{0}\right|^{-1} \sum_{w \in W_{0}}\left(w c_{+}\right)\left(-x_{0}\right)\left(w E\left(\cdot, \gamma_{\mu}\right)\right)\left(-x_{0}\right) \\
& =\left|W_{0}\right|^{-1} c_{+}\left(-x_{0}\right) .
\end{aligned}
$$

This proves the proposition. 


\subsection{Quadratic norms}

Finally, we derive the quadratic norms for the symmetric Wilson polynomials, see [21, Theorem 7.4].

Theorem 6.12. For $\lambda \in \Lambda^{+}$,

$$
\left\langle E^{+}\left(\cdot, \gamma_{\lambda}\right), E^{+}\left(\cdot, \gamma_{\lambda}\right)\right\rangle_{\mathbf{t}}^{+}=\frac{1}{N_{+}\left(\gamma_{\lambda}\right)},
$$

where

$$
N_{+}\left(\gamma_{\lambda}\right)=\frac{1}{\langle 1,1\rangle_{\mathbf{t}}^{+}} \frac{c_{+}^{\sigma}\left(-\gamma_{\lambda}\right)}{c_{+}^{\sigma}\left(-\gamma_{0}\right)} \prod_{\alpha \in \mathcal{R}_{r}^{+} \cap \tau(\lambda) \mathcal{R}_{r}^{-}} \frac{c_{-\alpha}^{\sigma}\left(\gamma_{\lambda}\right)}{c_{\alpha}^{\sigma}\left(\gamma_{\lambda}\right)}
$$

Proof. From (6.4) and Theorem 5.11 we obtain

$$
\begin{aligned}
\left\langle E^{+}\left(\cdot, \gamma_{\lambda}\right), E^{+}\left(\cdot, \gamma_{\lambda}\right)\right\rangle_{\mathbf{t}}^{+} & =\frac{\left|W_{0}\right|}{c_{+}\left(-x_{0}\right)}\left\langle E^{+}\left(\cdot, \gamma_{\lambda}\right), E\left(\cdot, \gamma_{\lambda}\right)\right\rangle_{\mathbf{t}} \\
& =\frac{\left|W_{0}\right| d_{\lambda, \lambda}}{c_{+}\left(-x_{0}\right) N\left(\gamma_{\lambda}\right)},
\end{aligned}
$$

where $d_{\lambda, \lambda}$ is the coefficient of $E\left(\cdot, \gamma_{\lambda}\right)$ in the expansion of $E^{+}\left(\cdot, \gamma_{\lambda}\right)$ from Proposition 6.1, i.e.,

$$
d_{\lambda, \lambda}=\frac{c_{+}^{\sigma}\left(-\gamma_{\lambda}\right)}{c_{+}^{\sigma}\left(-\gamma_{0}\right)}
$$

Writing out $N\left(\gamma_{\lambda}\right)$ and using Lemma 6.4 gives the result.

Open Access. This article is distributed under the terms of the Creative Commons Attribution Noncommercial License which permits any noncommercial use, distribution, and reproduction in any medium, provided the original author(s) and source are credited.

\section{References}

[1] Cherednik, I.: Inverse Harish-Chandra transform and difference operators. Int. Math. Res. Not. 1997(15), 733-750 (1997)

[2] Cherednik, I.: Double affine Hecke algebras. London Mathematical Society Lecture Note Series, vol. 319. Cambridge University Press, Cambridge (2005)

[3] Etingof, P., Gan, W.L., Oblomkov, A.: Generalized double affine Hecke algebras of higher rank. J. Reine Angew. Math. 600, 177-201 (2006)

[4] Etingof, P., Ginzburg, V.: Symplectic reflection algebras, Calogero-Moser space, and deformed Harish-Chandra homomorphism. Invent. Math. 147(2), 243-348 (2002)

[5] Etingof, P., Rains, E.: New deformations of group algebras of Coxeter groups. Int. Math. Res. Not. 2005(10), 635-646 (2005) 
[6] Etingof, P., Rains, E.: New deformations of group algebras of Coxeter groups, II. Geom. Funct. Anal. 17(6), 1851-1871 (2008)

[7] Gan, W.L., Ginzburg, V.: Deformed preprojective algebras and symplectic reflection algebras for wreath products. J. Algebra 283(1), 350-363 (2005)

[8] Groenevelt, W.: Fourier transforms related to a root system of rank 1. Transform. Groups 12(1), 77-116 (2007)

[9] Gustafson, R.A.: A generalization of Selberg's beta integral. Bull. Am. Math. Soc. (N.S.) 22(1), 97-105 (1990)

[10] Heckman, G.J.: Root systems and hypergeometric functions, II. Compositio Math. 64(3), 353-373 (1987)

[11] Koornwinder, T.H.: Askey-Wilson polynomials for root systems of type $B C$. Contemp. Math. 138, 189-204 (1992)

[12] Macdonald, I.G.: Affine Hecke algebras and orthogonal polynomials. Cambridge Tracts in Mathematics, vol. 157. Cambridge University Press, Cambridge (2003)

[13] Nishino, A., Ujino, H., Komori, Y., Wadati, M.: Rodrigues formulas for the non-symmetric multivariable polynomials associated with the $B C_{N}$-type root system. Nucl. Phys. B 571(3), 632-648 (2000)

[14] Noumi, M.: Macdonald-Koornwinder polynomials and affine Hecke rings. Surikaisekikenkyusho Kokyuroku 919, 44-55 (in Japanese) (1995)

[15] Opdam, E.M.: Harmonic analysis for certain representations of graded Hecke algebras. Acta Math. 175(1), 75-121 (1995)

[16] Sahi, S.: Nonsymmetric Koornwinder polynomials and duality. Ann. Math. (2) 150(1), 267-282 (1999)

[17] Sahi, S.: Some properties of Koornwinder polynomials, $q$-series from a contemporary perspective (South Hadley, MA, 1998), pp. 395-411. Contemp. Math., vol. 254. American Mathematical Society, Providence (2000)

[18] Stokman, J.V.: Koornwinder polynomials and affine Hecke algebras. Int. Math. Res. Not. 2000(19), 1005-1042 (2000)

[19] Stokman, J.V., Koornwinder, T.H.: Limit transitions for $B C$ type multivariable orthogonal polynomials. Can. J. Math. 49(2), 373-404 (1997)

[20] van Diejen, J.F.: Multivariable continuous Hahn and Wilson polynomials related to integrable difference systems. J. Phys. A 28(13), L369-L374 (1995)

[21] van Diejen, J.F.: Properties of some families of hypergeometric orthogonal polynomials in several variables. Trans. Am. Math. Soc. 351(1), 233-270 (1999)

[22] Wilson, J.A.: Some hypergeometric orthogonal polynomials. SIAM J. Math. Anal. 11(4), 690-701 (1980)

[23] Zhang, G.: Spherical transform and Jacobi polynomials on root systems of type BC. Int. Math. Res. Not. 2005(51), 3169-3189 (2005) 
Wolter Groenevelt

Delft Institute of Applied Mathematics

Technische Universiteit Delft

P.O. Box 5031

2600 GA Delft

The Netherlands

e-mail: W.G.M.Groenevelt@tudelft.nl 